Rotation numbers for quasi-periodically forced monotone circle maps

Stark, J and Feudel, $U$ and Glendinning, $\mathrm{P} A$ and Pikovsky, A

2002

MIMS EPrint: 2006.93

Manchester Institute for Mathematical Sciences

School of Mathematics

The University of Manchester

\footnotetext{
Reports available from: http://eprints.maths.manchester.ac.uk/

And by contacting: The MIMS Secretary

School of Mathematics

The University of Manchester

Manchester, M13 9PL, UK
} 


\title{
ROTATION NUMBERS FOR QUASI-PERIODICALLY FORCED MONOTONE CirCle MaPS
}

\author{
J. Stark ${ }^{1}$, U. Feudel ${ }^{2}$, P. Glendinning ${ }^{3}$ and A. Pikovsky ${ }^{2}$ \\ ${ }^{1}$ Centre for Nonlinear Dynamics and its Applications, University College \\ London, Gower Street, London, WC1E 6BT, UK. \\ 2 Institute of Theoretical Physics and Astrophysics, University of Potsdam, \\ Am Neuen Palais, PF 601553, D-14415 Potsdam, Germany. \\ ${ }^{3}$ Department of Mathematics, University of Manchester Institute of Sci- \\ ence and Technology, PO Box 88, Manchester, M60 1QD, UK.
}

Friday, October 27, 2000.

\begin{abstract}
Rotation numbers have played a central role in the study of (unforced) monotone circle maps. In such a case it is possible to obtain a priori bounds of the form $\rho-\frac{1}{n} \leq \frac{1}{n}\left(y_{n}-y_{0}\right) \leq \rho+\frac{1}{n}$, where $\frac{1}{n}\left(y_{n}-y_{0}\right)$ is an estimate of the rotation number obtained from an orbit of length $n$ with initial condition $y_{0}$, and $\rho$ is the true rotation number. This allows rotation numbers to be computed reliably and efficiently. Although Herman has proved that quasi-periodically forced circle maps also possess a well defined rotation number, independent of initial condition, the analogous bound does not appear to hold. In particular, two of the authors have recently given numerical evidence that there exist quasi-periodically forced circle maps for which $y_{n}-y_{0}-\rho n$ is not bounded. This renders the estimation of rotation numbers for quasiperiodically forced circle maps much more problematical. In this paper, we derive a new characterization of the rotation number for quasi-periodically forced circle maps based upon integrating iterates of an arbitrary smooth curve. This satisfies analogous bounds to above and permits us to develop improved numerical techniques for computing the rotation number. Additionally, we consider the boundedness of $y_{n}-y_{0}-\rho n$. We show that if this quantity is bounded (both above and below) for one orbit, then it is bounded for all orbits. Conversely, if for any orbit $y_{n}-y_{0}-\rho n$ is unbounded either above or below, then there is a residual set of orbits for which $y_{n}-y_{0}-\rho n$ is unbounded both above and below. In proving these results we also present a min-max characterization of the rotation number. We evaluate the performance of an algorithm based on this, and on the whole find it to be inferior to the integral based method.
\end{abstract}




\section{INTRODUCTION}

\subsection{PrinCiPAl Aims AND Results}

Many applications of nonlinear dynamics involve forced systems. Whilst forcing by a single frequency has long been extensively studied, that by two (or more) incommensurate frequencies has received comparatively less attention and is in general much more poorly understood. The simplest framework in which this can be studied is that of skew products over a rigid irrational rotation, that is maps of the form

$$
\begin{array}{llll}
\theta_{n+1} & = & \theta_{n}+\omega & \bmod 1 \\
y_{n+1} & = & g\left(\theta_{n}, y_{n}\right) &
\end{array}
$$

Here $\theta_{n} \in \mathbb{T}^{1}$, the unit circle and $\omega$ is irrational, whilst $y_{n}$ is assumed to lie in some finite dimensional manifold $Y$ (which in this paper will always be one dimensional). It will be convenient to write

$$
f(\theta) \quad=\quad \theta+\omega \quad \bmod 1
$$

and

$$
F(\theta, y)=(f(\theta), g(\theta, y))
$$

for the overall skew product map on $\mathbb{T}^{1} \times Y$. We shall furthermore define $g^{(n)}$ by $F^{n}(\theta, y)=$ $\left(f^{n}(\theta), g^{(n)}(\theta, y)\right)$.

One particularly interesting class of such systems is given by quasi-periodically forced circle maps, which correspond to $Y=\mathbb{T}^{1}$ (e.g. [Ding et al., 1989a; 1989b; Feudel et al., 1995; 1997; Chastell et al., 1995; Sturman, 1999; Glendinning et al., 2000]). It is usually more convenient to work with the lift (to $Y=\mathbb{R}$ ) of such a map; this is given by a $g: \mathbb{T}^{1} \times \mathbb{R} \rightarrow \mathbb{R}$ which satisfies $g(\theta, y+1)=g(\theta, y)+1$. This gives rise to a circle map in the usual way by taking $\tilde{g}: \mathbb{T}^{1} \times \mathbb{T}^{1} \rightarrow \mathbb{T}^{1}$ to be $\tilde{g}(\theta, y)=g(\theta, y) \bmod 1$. Then $(f, \tilde{g})$ is a map of the 2-torus $\mathbb{T}^{2}=\mathbb{T}^{1} \times \mathbb{T}^{1}$. In this paper we shall additionally restrict ourselves to the case where $(f, \widetilde{g})$ is a homeomorphism (a continuous invertible map with a continuous inverse) which in turns implies that $\widetilde{g}=(\theta, \bullet): \mathbb{T}^{1} \rightarrow \mathbb{T}^{1}$ is a homeomorphism of $\mathbb{T}^{1}$ for each $\theta \in \mathbb{T}^{1}$, and that $g$ is continuous in $\theta$. One would expect such maps to play as central a role for quasi-periodically forced systems as circle maps do in the class of general systems. A paradigm example (e.g. [Ding et al., 1989a; 1989b; Feudel et al., 1995; 1997; Chastell et al., 1995; Sturman, 1999; Glendinning et al., 2000]), comparable to the Arnold sine map ( $\mathrm{eg}$ [Arnold, 1957; 1983]), is given by

$$
g(\theta, y)=\quad=\quad y+\Omega+\frac{A}{2 \pi} \sin 2 \pi y+B \sin 2 \pi \theta
$$

This is a homeomorphism as long as $|A| \leq 1$. The condition that $\tilde{g}$ is a homeomorphism can be expressed in terms of the lift $g$ as a requirement that $g$ be strictly monotone, that is $g(\theta, y)$ $<g\left(\theta, y^{\prime}\right)$ for all $y<y^{\prime}$ and all $\theta \in \mathbb{T}^{1}$. In fact all of the results in this paper hold without the strictness assumption and we therefore define 
Definition 1.1 A quasi-periodically forced map $(f, g)$ on $\mathbb{T}^{1} \times \mathbb{R}$ is monotone if for all $y \leq y^{\prime}$ and all $\theta \in \mathbb{T}^{1}$

$$
g(\theta, y) \leq g\left(\theta, y^{\prime}\right)
$$

This property will turn out to play a central role in our work. Note that by induction we trivially have $g^{(n)}(\theta, y) \leq g^{(n)}\left(\theta, y^{\prime}\right)$ for all $n \in \mathbb{N}$, all $y \leq y^{\prime}$ and all $\theta \in \mathbb{T}^{1}$.

Monotonicity is of fundamental importance in the study of unforced circle maps and we shall therefore first review its consequences in this context. Thus, suppose that $h: \mathbb{R} \rightarrow \mathbb{R}$ is the lift of a monotone map of the circle, so that it satisfies $h(y+1)=h(y)+1$ and $h(y)<h\left(y^{\prime}\right)$ for all $y<y^{\prime}$. Then it has a unique rotation number defined by

$$
\rho \quad=\quad \lim _{n \rightarrow \infty} \frac{h^{n}(y)-y}{n}
$$

which is independent of the choice of $y$. This in fact turns out to still be the case if $h$ is not continuous; it is the monotonicity which ensures the existence and uniqueness of $\rho$ [Rhodes and Thompson, 1986; 1991].

The rotation number can be used to classify monotone circle maps and to organize their bifurcation diagram. Of particular interest are those regions for which $\rho$ is rational. For the classic Arnold sine map

$$
h(y) \quad=\quad=\quad \Omega+\frac{A}{2 \pi} \sin 2 \pi y
$$

these have a well known "tongue" shape and are usually referred to as Arnold tongues. Note that if $h$ has rotation number $p / q$ then $h^{q}$ - $p$ has rotation number 0 , and hence the $\rho=0$ tongue serves as an archetype for all the others.

A natural question to ask is how to compute the rotation number for a particular map. A naive approach is simply to take an initial point $y_{0}$, iterate it some large number $n$ times and then compute $\frac{1}{n}\left(h^{n}\left(y_{0}\right)-y_{0}\right)$. This turns out to work surprisingly well, largely due to the following bounds, which can easily be derived for all monotone circle maps [Rhodes and Thompson, 1986; 1991]:

$$
\rho-\frac{1}{n} \quad \leq \quad \frac{h^{n}(y)-y}{n} \leq \rho+\frac{1}{n}
$$

for all $y$. This can also be expressed as

$$
\left|\left(h^{n}(y)-y\right)-\rho n\right| \leq 1
$$

This bound gives tight control of how the rotation of orbits around the circle fluctuates about its average. One immediate consequence is that if $\rho=0$ then every orbit $y_{n}=h^{n}\left(y_{0}\right)$ is bounded and in particular must remain in the region $\left|y_{n}-y_{0}\right| \leq 1$. This can be used to provide rigorous error bounds in the numerical estimation of $\rho$. Thus, for instance, in estimating the extent of the $\rho=0$ tongue, we can immediately exclude parameter regions where $\left|h^{n}(0)\right|>1$ for any $n$. Furthermore, if $\rho \neq 0$, then we are guaranteed that $\left|h^{n}(0)\right|>1$ for all 
sufficiently large $n$. Similar statements, using $h^{n q}(0)-p$ instead of $h^{n}(0)$ apply to the general $p / q$ tongue. Finally, another immediate consequence of (1.6) is the continuity of $\rho$ with respect to parameters for continuous $h$.

Our main aim in this paper is to see to what extent these results can be generalized to the quasi-periodically forced case. We now have two rotation numbers, one in the $\theta$ direction and the other in the $Y$ direction. The former is trivially equal to $\omega$ and will not concern us further. The latter is defined by

$$
\rho \quad=\quad \lim _{n \rightarrow \infty} \frac{g^{(n)}(\theta, y)-y}{n}
$$

whenever the limit exists. The principal difficulty is that we no longer have ordering for arbitrary pairs of points. In other words, if $\theta \neq \theta^{\prime}$, then we have no information about the relative position of $g^{(n)}(\theta, y)$ and $g^{(n)}\left(\theta^{\prime}, y^{\prime}\right)$. This makes it impossible to apply the standard proof for the existence of a rotation number in the unforced case. Nevertheless, Herman shows that the monotonicity in each fibre, combined with the unique ergodicity of $f$ are sufficient to prove

Theorem 1.1 [Herman, 1983] If $F$ is a homeomorphism of the torus $\mathbb{T}^{1} \times \mathbb{T}^{1}$ of the form (1.2), then the limit (1.8) exists for all $(\theta, y)$, and is independent of $(\theta, y)$. Furthermore, the limit converges uniformly in $(\theta, y)$.

Thus, as before, one can talk of the rotation map of the map $F$. We shall give an elementary proof of this theorem as a corollary of our work below in \$3.3. However, it no longer appears possible to derive bounds analogous to (1.6) in the quasiperiodic case (see $\$ 1.2$ below). This makes the numerical estimation of $\rho$ by computing $\tilde{\rho}_{n}=\frac{1}{n}\left(g^{(n)}\left(\theta_{0}, y_{0}\right)-y_{0}\right)$ for a single initial $\left(\theta_{0}, y_{0}\right)$ potentially inaccurate and certainly fraught with uncertainty. This is illustrated in Figure 1.1 where we see that indeed (1.6) is not satisfied, and that large oscillations occur in $\tilde{\rho}_{n}$ as $n$ grows. These make it difficult to estimate the limit of $\tilde{\rho}_{n}$. One obvious improvement is to average over a number of different initial conditions, shown in Figure 1.2. Whilst this leads to a more accurate result, we see that it does not reduce the oscillations making it difficult both to estimate the limiting value, and the accuracy of our estimates.

Instead, in this paper we present a new integral characterization of the rotation number, for which we are able to derive a uniform estimate analogous to (1.6). This allows us to devise a family of algorithms for numerically estimating $\rho$ for which we can obtain explicit error bounds, exactly as in the unforced case. Furthermore, it turns out that in many circumstances such algorithms have superior performance to the simplistic ones outlined above. To explain our characterization, we begin by examining how curves are iterated under maps of the form (1.2):

Definition 1.2 The graph of a continuous function $\psi: \mathbb{T}^{1} \rightarrow \mathbb{R}$ is the set

$$
\text { graph } \psi=\left\{(\theta, \psi(\theta)): \theta \in \mathbb{T}^{1}\right\}
$$




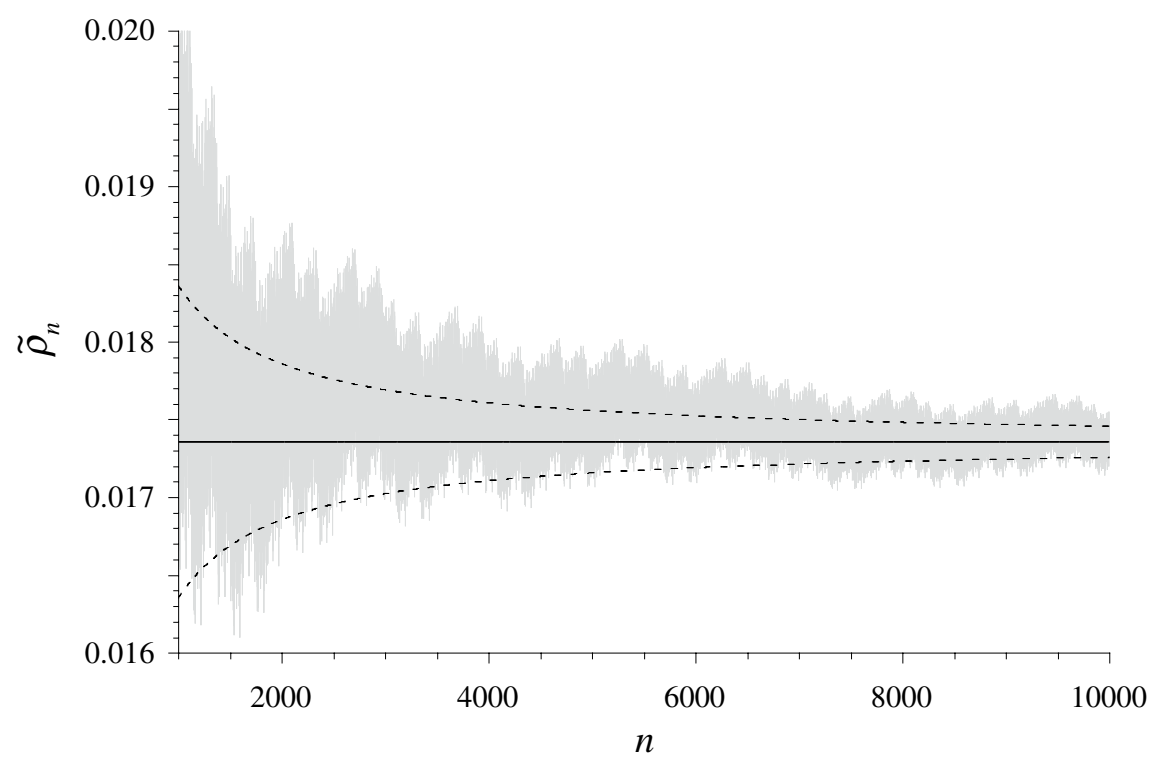

Figure 1.1. Estimate of rotation number for the map (1.3), with parameter values $A=0.8, B=$ 3.0 and $\Omega=0.01$, obtained by iterating a single randomly chosen orbit for $n$ iterations and computing $\tilde{\rho}_{n}=\frac{1}{n}\left(g^{(n)}\left(\theta_{0}, y_{0}\right)-y_{0}\right)$. The solid line indicates an accurate estimate $\rho^{*}=0.0173598$ of the limit value, obtained using Algorithm 1.4 (described below, with $k=1000$ points, a transient of $m=1000$, and $n=10^{5}$ iterates). The dashed line give the bounds $\rho^{*} \pm \frac{1}{n}$ analogous to (1.6).

Note that this is a curve winding once around the cylinder $\mathbb{T}^{1} \times \mathbb{R}$. For $n \in \mathbb{N}$, let $\psi_{n}: \mathbb{T}^{1} \rightarrow \mathbb{R}$ be the function whose graph is $F^{n}$ (graph $\psi$ ):

$$
\psi_{n}(\theta)=g^{(n)}(\theta, \psi(\theta))
$$

Our principal result is then

Theorem 1.2 If $F$ is a monotone continuous quasi-periodically forced circle map and $\psi_{n}$ is defined as above then

$$
\rho-\frac{1}{n} \quad \leq \quad \frac{1}{n} \int \psi_{n}-\psi d \theta \quad \leq \quad \rho+\frac{1}{n}
$$

Observe the close analogy of this to the fundamental bound (1.6) for the unforced case. A proof of this theorem given in $\$ 3$ below.

Remark 1.1 Those familiar with the theory of skew products should note that $\psi_{n}$ is not the standard graph transform of $\psi$, which is given by $\widetilde{\psi}_{n}(\theta)=g^{(n)}\left(f^{-n}(\theta), \psi\left(f^{-n}(\theta)\right)\right)$. The latter gives the $y$ co-ordinate of $F^{n}\left(\right.$ graph $\psi$ ) at $\theta$, whilst $\psi_{n}(\theta)$ gives the co-ordinate at $f^{n}(\theta)$ (Figure 1.3). Since Lebesque measure is invariant under $f$, we trivially have

$$
\int \varphi \circ f^{-n} d \theta \quad=\quad \int \varphi d \theta
$$



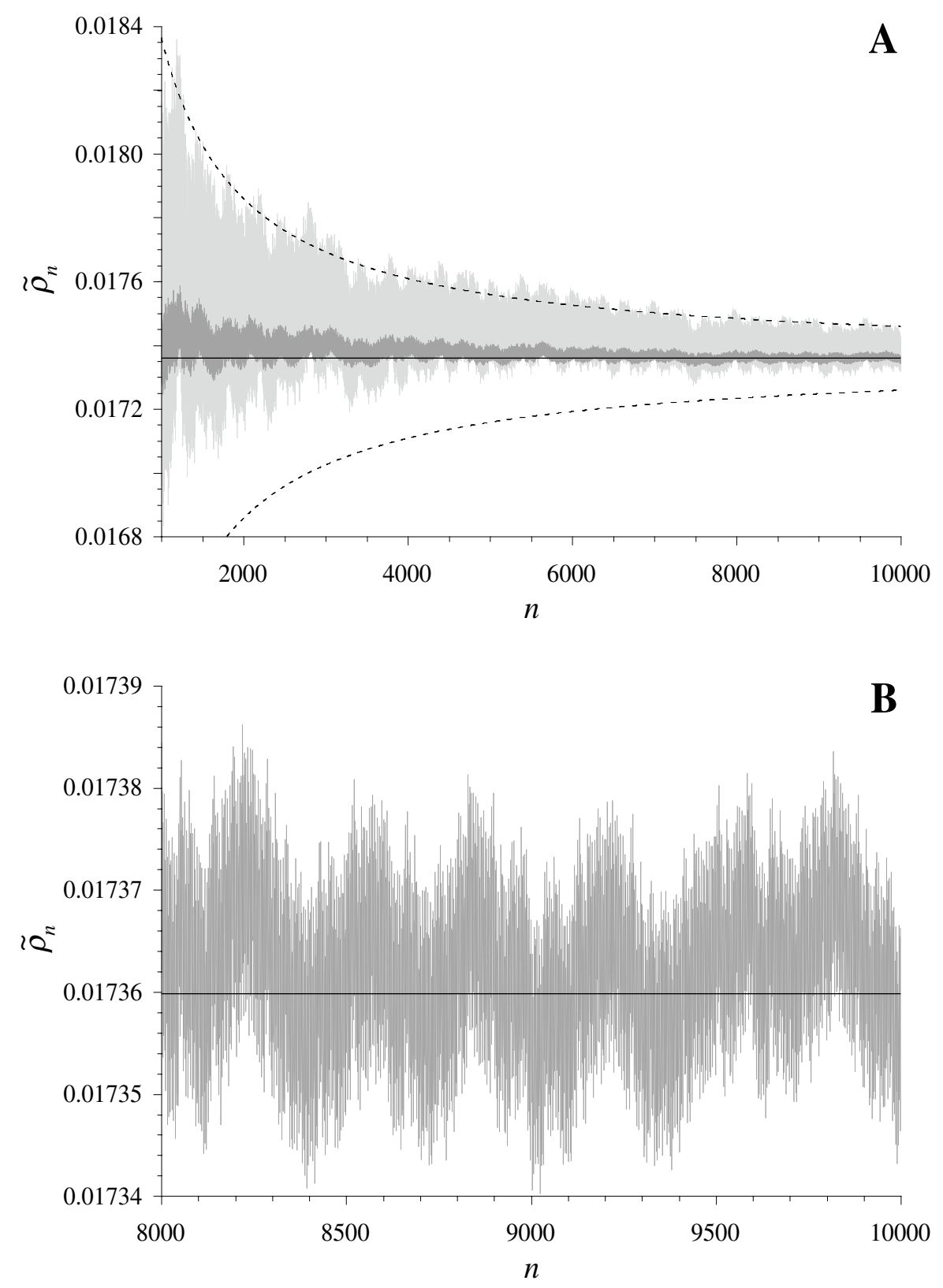

Figure 1.2. Estimate of rotation number for the same map as Figure 1.1. A) Average calculated using 10 (light grey) and 100 (dark grey) randomly chosen orbits. The limit value (solid line) and bounds (dashed line) are as in Figure 1.1. B) Detail of A, showing continuing oscillations in the estimate. Only the data for the average of 100 orbits is shown.

for any integrable $\varphi: \mathbb{T}^{1} \rightarrow \mathbb{R}$ and any $n \in \mathbb{Z}$. Hence in (1.9) we can replace $\psi_{n}$ by the graph transform $\tilde{\psi}_{n}$. In fact we can replace the integrand on the right hand side of (1.10) by $\varphi \circ R_{\alpha}$, for any $\alpha \in \mathbb{R}$, where $R_{\alpha}: \mathbb{T}^{1} \rightarrow \mathbb{T}^{1}$ is the rigid rotation $R_{\alpha}(\theta)=\theta+\alpha \bmod 1$. 


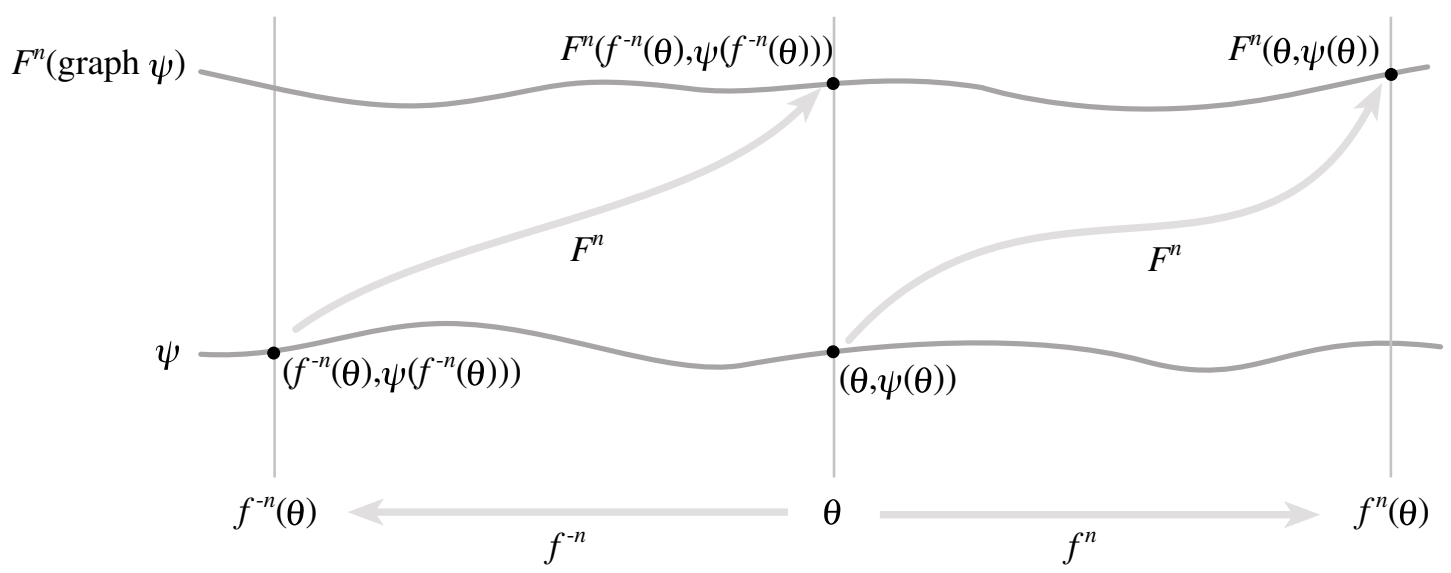

Figure 1.3 The function $\psi_{n}=g^{(n)}(\theta, \psi(\theta))$ and the graph transform $\widetilde{\psi}_{n}(\theta)=g^{(n)}\left(f^{-n}(\theta), \psi\left(f^{-n}(\theta)\right)\right)$.

\subsection{BOUNDED V. UNBOUNDED ORBITS}

Recall that for an unforced circle map of rotation number $\rho$, the quantity $\rho n$ provides a very tight estimate of how far an orbit has moved in $n$ iterations. In particular (1.7) implies that $h^{n}(y)-y-\rho n$ is bounded. Unfortunately, it seems that this is no longer true in the quasiperiodically forced case, and in particular [Feudel et al., 1995] present strong numerical evidence that there exist parameter values for the map (1.3) where $y_{n}$ grows approximately logarithmically. This is thus an example of a map for which $\rho=0$ but $g^{(n)}(\theta, y)-y$ is not bounded. It turns out that in general if this occurs for one orbit, then it occurs for all typical orbits. Conversely if there exists an orbit for which $g^{(n)}\left(\theta_{0}, y_{0}\right)-y$ is bounded, then $g^{(n)}(\theta, y)-y$ is uniformly bounded for all $(\theta, y) \in \mathbb{T}^{1} \times \mathbb{R}$. An appropriate generalization holds for other rotation numbers. Thus define:

Definition 1.3 We say that the orbit of $(\theta, y)$ is $\rho$-bounded above if there exists a constant $K$ such that $g^{(n)}(\theta, y)-y-\rho n \leq K$ for all $n \in \mathbb{N}$ and $\rho$-bounded below if $g^{(n)}(\theta, y)-y-\rho n \geq-K$ for all $n \in$ $\mathbb{N}$. An orbit is $\rho$-bounded if it is both $\rho$-bounded above and below. It is $\rho$-unbounded if it is not $\rho$-bounded and similarly for $\rho$-unbounded above and $\rho$-unbounded below.

If there exists a $\rho$-bounded orbit, then the map has rotation number $\rho$, but the converse does not necessarily hold. In $\$ 4$ below, we prove:

Theorem 1.3 Suppose that a monotone continuous quasi-periodically forced circle map $F$ has rotation number $\rho$. Then there exists at least one orbit that is $\rho$-bounded above and one orbit that is $\rho$-bounded below. If there exists an orbit that is $\rho$-bounded (both above and below), then all orbits are $\rho$-bounded. If there is no $\rho$-bounded orbit then there exists a residual subset $U \subset \mathbb{T}^{1}$ such that all $(\theta, y) \in U \times \mathbb{R}$ are $\rho$-unbounded both above and below.

Numerical evidence suggests that the $\rho$-unbounded situation can occur [Feudel et al., 1995], however we know of no rigorous proof of this. In such a case the set $U$ can clearly be chosen to be $f$-invariant, and hence must be of either zero or full Lebesgue measure. The fact that 
all numerical orbits in the [Feudel et al., 1995] example appear to be unbounded suggests that $U$ in fact has full measure, though we can see no way of proving this.

As part of the proof of the above theorem we also derive the following "min-max" characterization of the rotation number, which motivates a number of numerical algorithms described in the next section.

Theorem 1.4 Suppose that a monotone continuous quasi-periodically forced circle map $F$ has rotation number $\rho$ and let $\psi: \mathbb{T}^{1} \rightarrow \mathbb{R}$ be an arbitrary function. Then

$$
\inf _{\theta \in \mathbb{T}^{1}} \sup _{n \in \mathbb{N}} \frac{1}{n}\left(g^{(n)}(\theta, \psi(\theta))-\psi(\theta)-1\right)=\rho \quad=\quad \sup _{\theta \in \mathbb{T}^{1}} \inf _{n \in \mathbb{N}} \frac{1}{n}\left(g^{(n)}(\theta, \psi(\theta))-\psi(\theta)+1\right)
$$

This is proved under the guise of Corollary 5.3 below.

\subsection{NuMERICAL METHODS FOR ESTIMATING THE Rotation NuMbER}

We consider the following methods for numerically computing the rotation number of a quasi-periodically forced circle map. The first two have been widely used in the literature [Feudel et al., 1995; 1997; Glendinning et al., 2000], whilst the remainder are motivated by the theoretical results described above. A numerical evaluation of these methods is given in the next section.

Algorithm 1.1 Choose an initial condition $\left(\theta_{0}, y_{0}\right) \in \mathbb{T}^{1} \times \mathbb{R}$ and some large $n \in \mathbb{N}$. Then an estimate for $\rho$ is given by (Figure 1.1)

$$
\tilde{\rho}_{1, n} \quad=\quad \frac{1}{n}\left(g^{(n)}\left(\theta_{0}, y_{0}\right)-y_{0}\right)
$$

Somewhat better results can be obtained by choosing many different initial conditions.

Algorithm 1.2 Choose a random set of initial conditions $\left\{\left(\theta_{0}, y_{0}\right), \ldots,\left(\theta_{k-1}, y_{k-1}\right)\right\} \subset \mathbb{T}^{1} \times \mathbb{R}$ and some large $n \in \mathbb{N}$. Estimate $\rho$ using (Figure 1.2)

$$
\tilde{\rho}_{2, n} \quad=\quad \frac{1}{k} \sum_{i=0}^{k-1} \frac{1}{n}\left(g^{(n)}\left(\theta_{i}, y_{i}\right)-y_{i}\right)
$$

The disadvantage of these methods is that we have no estimate of the error in determining $\rho$ for a particular choice of $n$ and $k$. Theorem 1.2 on the other hand offers error bounds that are explicit and apart from the inaccuracies involved in evaluating an integral also rigorous. It suggests the following.

Algorithm 1.3 Choose a smooth function $\psi: \mathbb{T}^{1} \rightarrow \mathbb{R}$; in the absence of $a$ priori information, we may as well take $\psi$ to be constant. Choose some $k \in \mathbb{N}$ and subdivide $\mathbb{T}^{1}$ into $k$ equally spaced intervals, so that $\theta_{i}=\frac{i}{k}$ for $i=0, \ldots, k$-1. Iterate each point $\left(\theta_{i}, \psi\left(\theta_{i}\right)\right)$ for $n$ iterations and estimate the integral in (1.9) using the trapezoidal rule. Thus:

$$
\tilde{\rho}_{3, n} \quad=\quad \frac{1}{k} \sum_{i=0}^{k-1} \frac{1}{n}\left(g^{(n)}\left(\theta_{i}, \psi\left(\theta_{i}\right)\right)-\psi\left(\theta_{i}\right)\right)
$$


Note the close similarity to Algorithm 1.2: the only difference is in the choice of initial points. Remarkably, not only does this lead to rigorous error bounds, but also appears to give a more accurate estimate of $\rho$.

We also remark that for periodic functions, the trapezoidal rule is about as accurate a method as one can get for the numerical evaluation of an integral [Davis and Rabinowitz, 1984], and hence there is no point attempting to use more sophisticated quadrature formulas. Of course, the error analysis of the trapezoidal rule assumes that the funciton to be integrated is smooth, and the higher the degree of smoothness, the higher the order of the error estimates. In our case, if $\psi$ and $g$ are smooth, then so will $\psi_{n}$ be. However, especially if the system possesses a strange non chaotic attractor, the derivatives of $\psi_{n}$ can grow without bound as $n \rightarrow \infty$ [Feudel et al., 1995]. Hence, in principle a numerical estimate of the integral of $\psi_{n}$ could be become quite inaccurate. In practice, however, this does not seem to be a problem, as we shall see when we evaluate this algorithm in the next section.

When the map $F$ has an invariant or periodic curve, it seems natural to use this as $\psi$ in the above scheme. Observe that if we knew the invariant curve precisely and used it for $\psi$ then we would have

$$
\int \psi_{n} d \theta=\int \psi d \theta
$$

and hence would have an exact evaluation of the rotation number (which of necessity is 0 when there is an invariant curve) for any $n$. Similarly if the curve was periodic, we would get an exact result for any $n$ which was a multiple of the period.

Unfortunately, we will in general not know the invariant curve $a$ priori. However, if it is attracting (and typically inside a phase-locked tongue an attracting curve will exist) we can approximate it by simply iterating an arbitrary curve for some initial number of iterations $m$. In effect we incorporate a transient in Algorithm 1.3 leading to

Algorithm 1.4 Choose a smooth function $\psi: \mathbb{T}^{1} \rightarrow \mathbb{R}$ and $k, m, n \in \mathbb{N}$. Subdivide $\mathbb{T}^{1}$ into $k$ equally spaced intervals, so that $\theta_{i}=\frac{i}{k}$ for $i=0, \ldots, k$-1. Iterate each point $\left(\theta_{i}, \psi\left(\theta_{i}\right)\right)$ for an initial transient of length $m$ and then for a further $n$ iterations. This gives the following estimate of the rotation number

$$
\tilde{\rho}_{4, n} \quad=\quad \frac{1}{k} \sum_{i=0}^{k-1} \frac{1}{n}\left(g^{(n+m)}\left(\theta_{i}, \psi\left(\theta_{i}\right)\right)-g^{(m)}\left(\theta_{i}, \psi\left(\theta_{i}\right)\right)\right.
$$

We now turn to algorithms motivated by Theorem 1.4, and in particular by Lemma 5.1:

Algorithm 1.5 Choose a smooth function $\psi: \mathbb{T}^{1} \rightarrow \mathbb{R}$ and $k, n \in \mathbb{N}$. Subdivide $\mathbb{T}^{1}$ into $k$ equally spaced intervals, so that $\theta_{i}=\frac{i}{k}$ for $i=1, \ldots, k$. Iterate each point $\left(\theta_{i}, \psi\left(\theta_{i}\right)\right) n$ times and set

$$
\begin{array}{lll}
\alpha_{n}\left(\theta_{i}\right) & = & \max _{1 \leq m \leq n} \frac{1}{m}\left(g^{(m)}\left(\theta_{i}, \psi\left(\theta_{i}\right)\right)-\psi\left(\theta_{i}\right)-1\right) \\
\alpha_{n}^{+}\left(\theta_{i}\right) & = & \min _{1 \leq m \leq n} \frac{1}{m}\left(g^{(m)}\left(\theta_{i}, \psi\left(\theta_{i}\right)\right)-\psi\left(\theta_{i}\right)+1\right)
\end{array}
$$




$$
\begin{array}{lll}
\dot{\alpha_{n}} & = & \min _{1 \leq i \leq k} \dot{\alpha}_{n}^{-}\left(\theta_{i}\right) \\
\alpha_{n}^{+} & = & \max _{1 \leq i \leq k} \alpha_{n}^{+}\left(\theta_{i}\right)
\end{array}
$$

As in Algorithm 1.4, we can also add an initial transient. Then apart from the effects of discretizing $\mathbb{T}^{1}$ (which is analogous to the discretization involved in evaluating the integrals in the previous two algorithms), we have

$$
\overline{\alpha_{n}} \leq \rho \leq \alpha_{n}^{+}
$$

Furthermore, Lemma 5.2 shows that as we increase the number of iterations, the width of the interval $\left[\alpha_{n}, \alpha_{n}^{+}\right]$shrinks to zero; that is

$$
\lim _{n \rightarrow \infty} \alpha_{n}^{-} \quad=\quad \rho \quad=\quad \lim _{n \rightarrow \infty} \alpha_{n}^{+}
$$

Finally we remark that often we do not actually want to estimate $\rho(F)$, but rather obtain bounds for it, for instance as part of a bracketing or root finding routine for the edges of a tongue. Since Theorem 1.2 provides a priori bounds for Algorithm 1.3 and Algorithm 1.4, it is easy to modify these for this purpose:

Algorithm 1.6 Choose a candidate $\rho$, a smooth function $\psi: \mathbb{T}^{1} \rightarrow \mathbb{R}$ and $k, m, n \in \mathbb{N}$. Subdivide $\mathbb{T}^{1}$ into $k$ equally spaced intervals, so that $\theta_{i}=\frac{i}{k}$ for $i=0, \ldots, k$-1. Iterate each point $\left(\theta_{i}, \psi\left(\theta_{i}\right)\right)$ for an initial transient of length $m$ and then for a maximum of $n$ iterations. If at any $j \leq n$ we have

$$
\frac{1}{k} \sum_{i=0}^{k-1} g^{(j+m)}\left(\theta_{i}, \psi\left(\theta_{i}\right)\right) \quad>\quad j \rho+1+\frac{1}{k} \sum_{i=0}^{k-1} g^{(m)}\left(\theta_{i}, \psi\left(\theta_{i}\right)\right)
$$

then $\rho(F)>\rho$, and similarly for an upper bound If we reach $n$ iterations without satisfying this inequality then the algorithm does not given a bound on $\rho(F)$. Of course in that case we can apply Algorithm 1.4 to estimate $\rho(F)$ without further work.

The advantage of this approach over simply iterating for the full $n$ iterates and then seeing whether $\tilde{\rho}_{4, n}>\rho+\frac{1}{n}$ is that when $\rho(F)$ is significantly larger than $\rho$, we can satisfy (1.11) with $j$ much less than $n$, and hence deduce that $\rho(F)>\rho$ with much less work. A min-max approach to the same problem is suggested by Lemma 4.5. This implies that if for each $\theta \in \mathbb{T}^{1}$ there exists a $m(\theta) \in \mathbb{N}$ such that $g^{(m(\theta)}(\theta, \psi(\theta))-\psi(\theta)-m(\theta) \rho>1$, then $\rho(F)>\rho$. Similarly if $g^{(m(\theta)}(\theta, \psi(\theta))-\psi(\theta)-m(\theta) \rho<-1$ for all $\theta \in \mathbb{T}^{1}$, then $\rho(F)<\rho$. We can also incorporate an initial transient. This leads to the following scheme

Algorithm 1.7 Choose a smooth function $\psi: \mathbb{T}^{1} \rightarrow \mathbb{R}$ and $k, m, n \in \mathbb{N}$. Subdivide $\mathbb{T}^{1}$ into $k$ equally spaced intervals, so that $\theta_{i}=\frac{i}{k}$ for $i=0, \ldots, k$-1. Iterate each point $\left(\theta_{i}, \psi\left(\theta_{i}\right)\right)$ for an initial transient of length $m$ and then for up to $n$ iterations. If at any $j \leq n$ we have $g^{(j+m)}\left(\theta_{i}, \psi\left(\theta_{i}\right)\right)$ $>j \rho+g^{(m)}\left(\theta_{i}, \psi\left(\theta_{i}\right)\right)+1$, stop and go on to the next point. If this happens for all $i=0, \ldots, k-1$, then $\rho(F)>\rho$. If for any $i$ we reach $n$ iterations $\left(i . e . g^{(j)}\left(\theta_{i}, \psi\left(\theta_{i}\right)\right) \leq j \rho+g^{(m)}\left(\theta_{i}, \psi\left(\theta_{i}\right)\right)+1\right.$ for all $j=1$, $\ldots, n)$ then stop; the algorithm fails to give a bound for $\rho(F)$ in this case. 


\section{NUMERICAL RESULTS}

Since most readers will probably be mainly interested in the algorithms for estimating the rotation number, we present an evaluation of these first, before giving rigorous proofs of the above theorems in subsequent sections. Our tests were performed using the map (1.3), initially focusing on the parameter values $A=0.8, B=3.0$ and $\Omega=0$ where [Feudel et al., 1995] suggest the system has a strange nonchaotic attractor with unbounded orbits (because of the symmetry in the map, $\Omega=0$ implies that $\rho=0$ and hence $\rho$-bounded and bounded are synonymous). This represents the most difficult situation possible for which to estimate a rotation number. If the map has a smooth invariant (or periodic) circle, then even Algorithm 1.1 can give an excellent estimate of the rotation number, particularly when $n$ is a convergent of $\omega$ (ie a Fibonacci number when $\omega$ is the golden mean). Even when the system has a strange nonchaotic attractor, but all orbits are bounded, then such bounds imply bounds on the convergence of (1.8) and hence of Algorithm 1.1. On the other hand when there exist $\rho$-unbounded orbits, then we expect (1.8) to converge particularly poorly.

Unfortunately, preliminary numerical investigations showed that because of the symmetry of the map at $\Omega=0$, some of the improvements in performance exhibited by Algorithm 1.3 were caused by subtle cancellations (due to the symmetry), and were thus to some extent spurious. We therefore broke the symmetry by setting $\Omega=0.01$, and this is the value for the numerical work presented here.

The disadvantage of the choice $\Omega=0.01$ is that we no longer know a priori the rotation number, unlike the case $\Omega=0$, where $\rho=0$. This makes it more difficult to evaluate the error in the various estimates of the rotation number. We overcame this by using a much larger number of iterations than used in the remainder of our work to compute an accurate estimate $\rho^{*} \approx 0.0173598 \ldots$. This was done using both Algorithm 1.3 and Algorithm 1.4, with $k=$ 1000 points, $n=10^{5}$ iterates, and a transient of $m=1000$ in the case of Algorithm 1.4. A curve of the form $\tilde{\rho}_{n}=\rho^{*}+c n^{-1}$ was then fitted to estimate $\rho^{*}$.

We have already presented results for Algorithm 1.1 and Algorithm 1.2, in Figure 1.1 and Figure 1.2 respectively. We see that increasing the number of orbits used improves the accuracy of the estimate, but does not reduce the oscillations in the convergence. We additionally tried using a transient, similar to that in Algorithm 1.4, in these algorithms but found that it led to no significant improvement (results not shown).

By contrast, using Algorithm 1.3 leads both to more accurate results, and to much more regular convergence (Figure 2.1). The convergence in the case of 1000 orbits is particularly smooth, making it very easy to fit a line of the form $\tilde{\rho}_{n}=\rho^{*}+c n^{-1}$ to estimate $\rho^{*}$, in contrast to Algorithm 1.2. On the other hand, increasing the number of orbits from 100 to 1000 actually makes little difference to accuracy. Note that the bounds (1.9) are satisfied even by Algorithm 1.2 when we take a large number of orbits. It would be interesting to see whether this will always be the case in the limit of taking a large number of points (ie $k \rightarrow \infty$ ).

Figure 2.2 shows the effect of including a transient. Whereas we found that this had little effect for Algorithm 1.2 (results not shown), it significantly appears to improve the accuracy 
of Algorithm 1.3, as shown in Figure 2.2. Increasing the length of the transient to $m=1000$ gave little significant improvement to the results (not shown). Figure 2.2B represents the best possible results we were able to achieve, and comparison to Figure 2.1B shows the dramatic improvement that is possible over a random choice of orbits.

Next, we turn to the algorithms based on the min-max characterization of the rotation number given by Theorem 1.4. Figure 2.3 shows that $\alpha_{n}^{-}$and $\alpha_{n}^{+}$provide reasonably tight bounds for the rotation number. Observe that unlike the previous algorithms, $\alpha_{n}^{-}$and $\alpha_{n}^{+}$
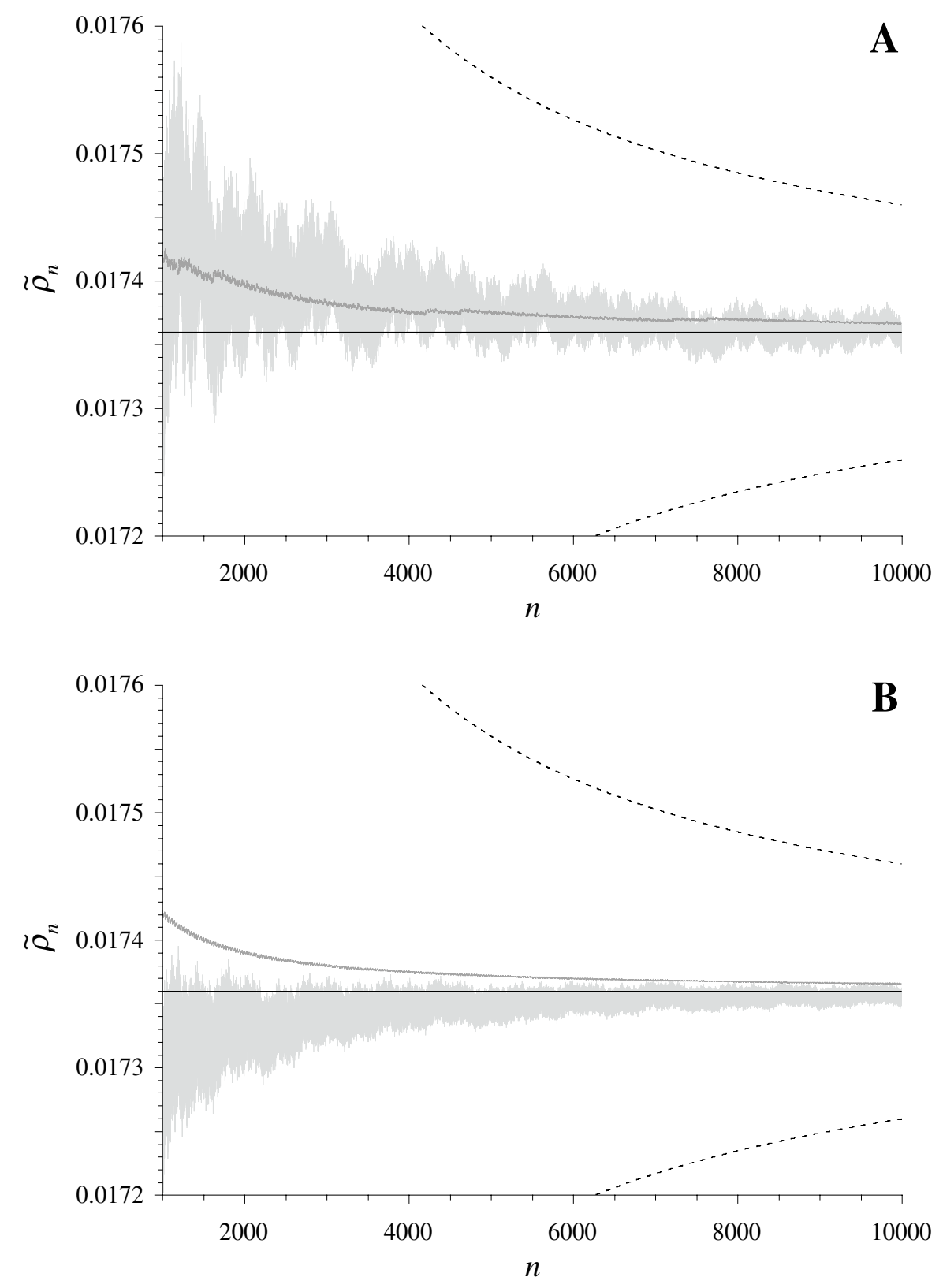

Figure 2.1. Comparison of Algorithm 1.2 (light grey) and Algorithm 1.3 (dark grey) for the same map as Figure 1.1, using A) 100 orbits and B) 1000 orbits. The solid line gives the estimate $\rho^{*}=0.0173598$ and the dashed line gives the bounds $\rho^{*} \pm \frac{1}{n}$, as in (1.9). 
converge to the rotation number in a monotone fashion, as is obvious from their definition. This definition also implies that increasing the number of orbits widens the interval within which we estimate the rotation number to be. We thus get a less accurate, but presumably more reliable estimate of the rotation number. This is apparent in Figure 2.3 and is again in contrast to the previous algorithms where using more points leads to improved accuracy. Including a transient in Algorithm 1.5 leads to some improvement in performance, but this is not dramatic (results not shown).
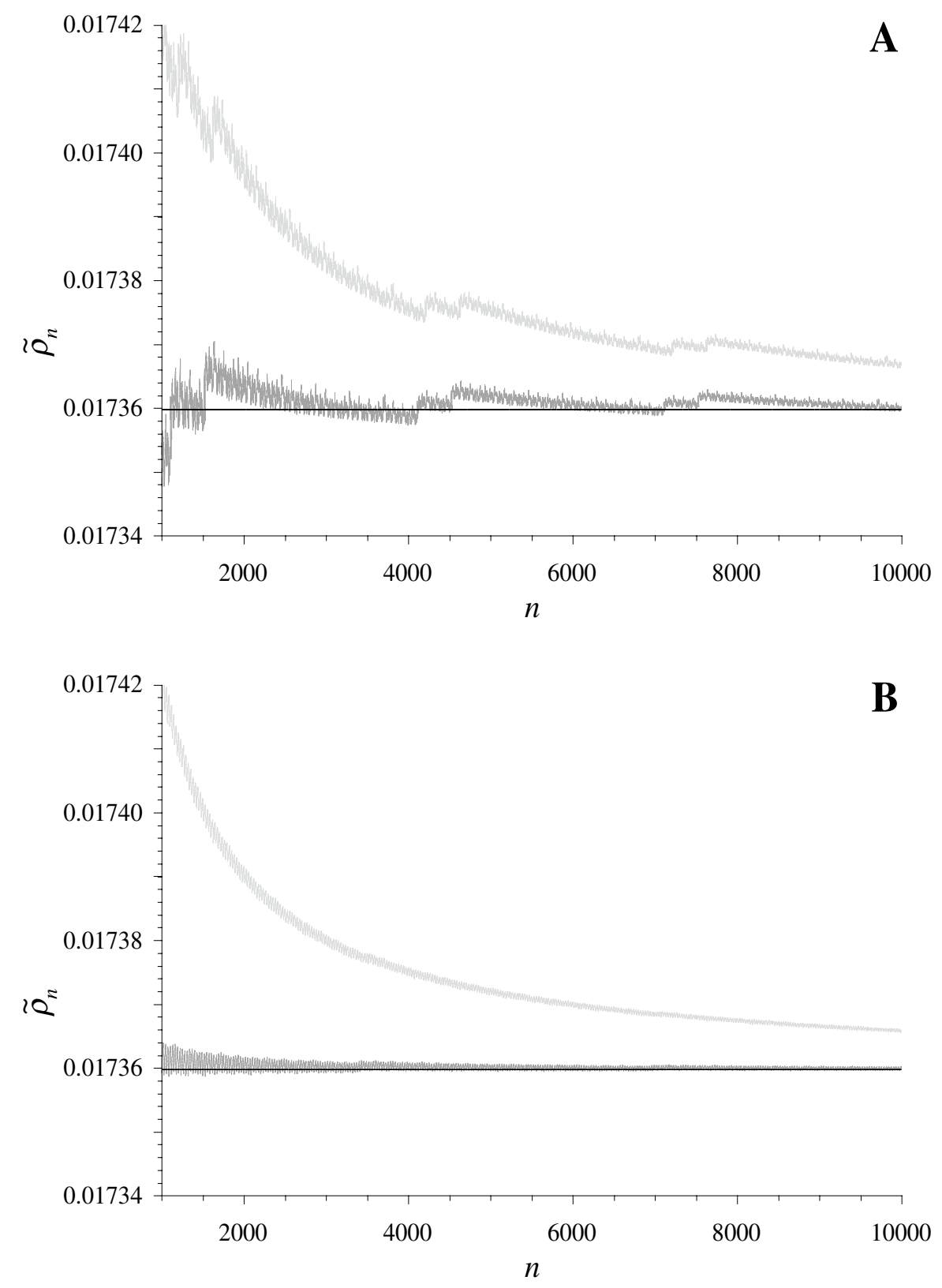

Figure 2.2. Effect of including a transient of length $m=100$ in Algorithm 1.3, ie a comparison between Algorithm 1.3 (light grey) and Algorithm 1.4 (dark grey) for the same map as Figure 1.1, using A) 100 orbits and B) 1000 orbits. The solid line gives the estimate $\rho^{*}=0.0173598$. 
The results of Algorithm 1.5 are not directly comparable with those of Algorithm 1.3, ie we cannot directly compare Figure 2.3 with Figure 2.1. This is because $\alpha_{n}^{ \pm}$are estimates of bounds for the rotation number, whilst $\tilde{\rho}_{3, n}$ is an estimate of the rotation number itself. The appropriate comparison is therefore between $\alpha_{n}^{ \pm}$and $\tilde{\rho}_{3, n} \pm \frac{1}{n}$. This is shown in Figure 2.4.

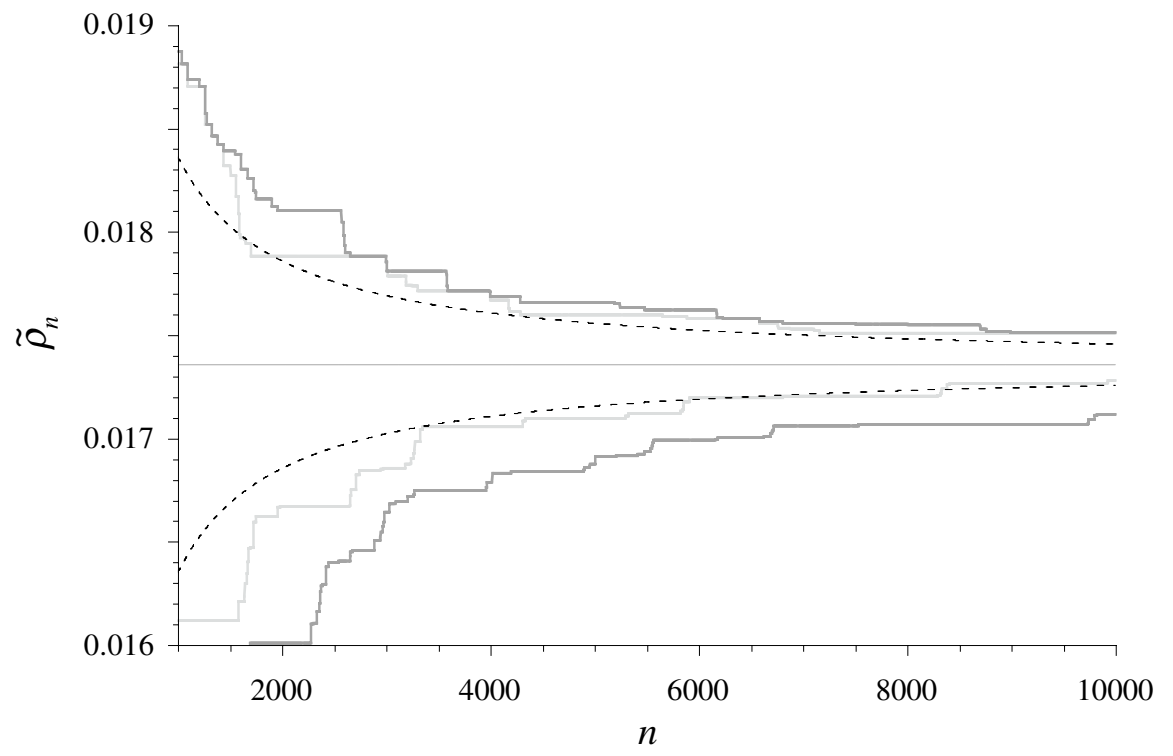

Figure 2.3. Evaluation of Algorithm 1.5 for the same map as Figure 1.1, using 100 orbits (light gray) and 1000 orbits (dark grey). The solid line gives the estimate $\rho^{*}=0.0173598$ and the dashed line gives the bounds $\rho^{*} \pm \frac{1}{n}$, as in (1.9).

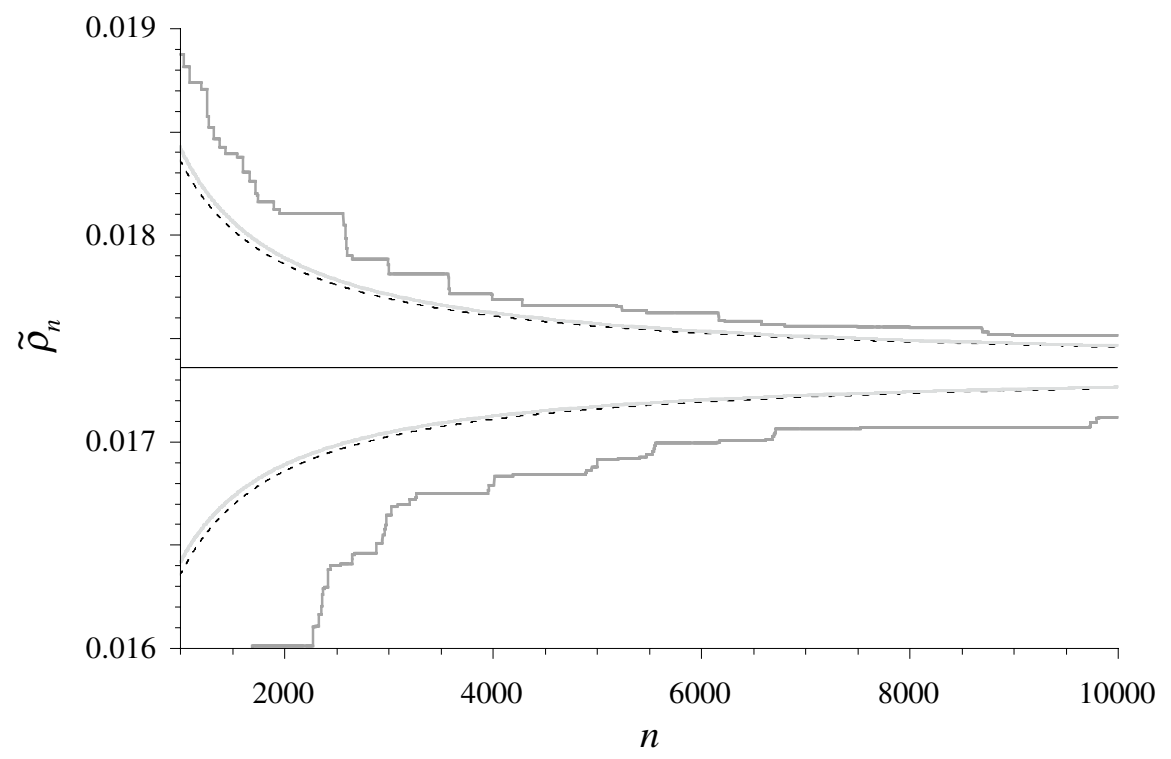

Figure 2.4. Comparison of the bounds derived from Algorithm 1.5 (dark grey, same data as in Figure 2.3) and those obtained from Algorithm 1.3 (light grey, derived from data in Figure 2.1B). In both case 1000 orbits with no transient were used. The bounds for Algorithm 1.3 were obtained by computing $\tilde{\rho}_{3, n} \pm \frac{1}{n}$. The solid line and dashed lines are as in Figure 2.3. 
We see that since $\tilde{\rho}_{3, n}$ is such a good estimate, the bounds $\tilde{\rho}_{3, n} \pm \frac{1}{n}$ are close to optimal, whilst those given by $\alpha_{n}^{ \pm}$are significantly wider. Furthermore, at least in our implementation, Algorithm 1.3 is about 1.5 times faster than Algorithm 1.5 and hence it appears that for the purposes of numerical estimation of the rotation number the integral representation given by Theorem 1.2 is superior to the min-max approach of Theorem 1.4.

The situation is reversed when we compare Algorithm 1.6 with Algorithm 1.7, where the min-max approach is much more efficient. This is illustrated in Figure 2.5 where we show the total number of iterations of the map (1.3) that each algorithm requires to show that $\rho(\Omega)>0$, as a function of $\Omega$, at the usual parameter values $A=0.8$ and $B=3.0$. Note that there is evidence [Feudel et al., 1995; Glendinning et al., 2000] that for these values of $A$ and $B$, the tongue $\rho=0$ has zero width, ie that $\rho(\Omega)>0$ for $\Omega>0$ and this is confirmed by Figure 2.5.

It is interesting that the two algorithms exhibit different scaling with $\Omega$. The relatively slow growth of $N_{\text {tot }}$ as $\Omega \rightarrow 0$ suggests that the computer only sees a few orbits that are $\rho$ unbounded below, in apparent contradiction to the remark after Theorem 1.3. This is similar to the apparent discrepancies between theory and numerical experiment observed in [Sturman, 2000]. Also observe that in the case of Algorithm 1.6, $1 / j=k / N_{\text {tot }}$ is a good estimate for $\rho(\Omega)$. Thus for $\Omega \leq 10^{-3}$, we have a very good fit to $\rho(\Omega) \sim \Omega^{-1}$. This is confirmed by estimating $\rho(\Omega)$ directly using Algorithm 1.4 with $k=1000, n=10^{5}, m=1000$, shown in Figure 2.6. This gives an almost perfect fit to $\rho(\Omega)=c \Omega^{-1}$ for $\Omega \leq 10^{-3}$, with $c \approx 6.37 \ldots$. Other reasonable choices of $k, n$ and $m$ yield identical results. It would interesting to see whether one can give a theoretical explanation of this.

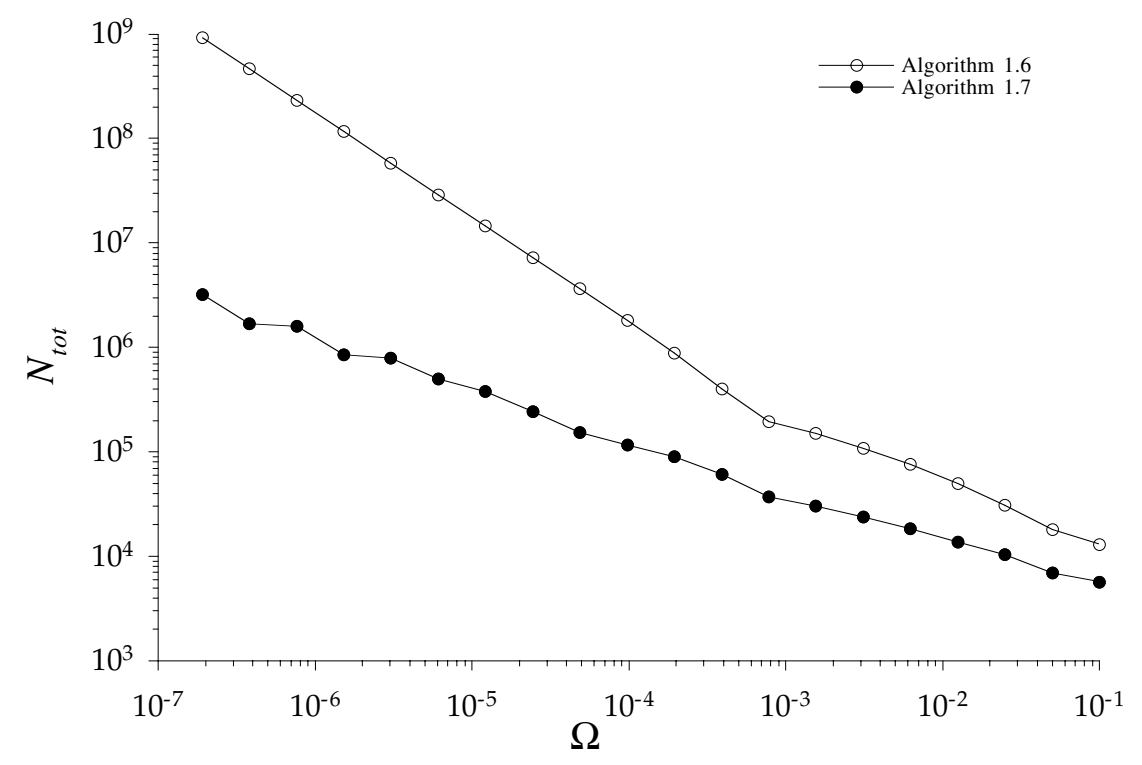

Figure 2.5. Comparison of Algorithm 1.6 and Algorithm 1.7. We show the total number of iterates $N_{\text {tot }}$ of map (1.3), as a function of $\Omega$ required to show that the rotation number is strictly positive, ie that we are not in the $\rho=0$ tongue. The other parameter values $A=0.8$ and $B=3.0$ are as before. In both cases, $k=1000$ orbits were used. For Algorithm 1.6, $N_{\text {tot }}=$ $j k$, where $j$ is the first iterate to satisfy (1.11), whilst for Algorithm 1.7, $N_{\text {tot }}=j(1)+j(1)+\ldots+$ $j(k)$, where $j(i)$ is the first iterate such that $g^{(j(i)+m)}\left(\theta_{i}, \psi\left(\theta_{i}\right)\right)>g^{(m)}\left(\theta_{i}, \psi\left(\theta_{i}\right)\right)+1$. 


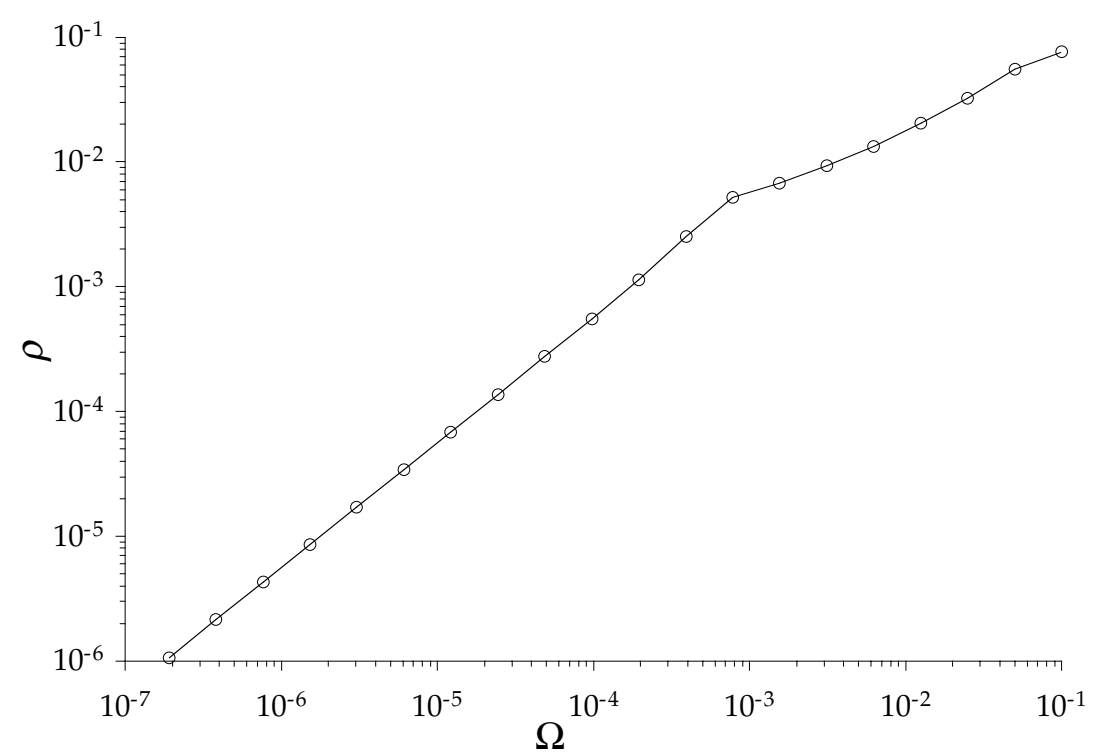

Figure 2.6. Estimate of the rotation number $\rho(\Omega)$ of map (1.3), as a function of $\Omega$ with $A=0.8$ and $B=3.0$, using Algorithm 1.4 with $k=1000, n=10^{5}, m=1000$.

\section{Proof of Theorem 1.2}

\subsection{ELEMENTARY RESULTS}

We begin with a number of elementary results, which are identical to their analogues for unforced circle maps. Recall that we define $g^{(n)}$ by $F^{n}(\theta, y)=\left(f^{n}(\theta), g^{(n)}(\theta, y)\right)$.

Lemma 3.1 If $F$ is a skew product of the form (1.2), then $g^{(n+m)}(\theta, y)=g^{(m)}\left(f^{n}(\theta), g^{(n)}(\theta, y)\right)$ for all $n, m \in \mathbb{N}$ and all $(\theta, y) \in \mathbb{T}^{l} \times \mathbb{R}$.

Proof By definition we have $\left(f^{n+m}(\theta), g^{(n+m)}(\theta, y)\right)=F^{n+m}(\theta, y)=F^{m}\left(f^{n}(\theta), g^{(n)}(\theta, y)\right)=\left(f^{m}\left(f^{n}(\theta)\right)\right.$, $\left.g^{(m)}\left(f^{n}(\theta), g^{(n)}(\theta, y)\right)\right)$. Matching the second co-ordinates gives the required result.

Corollary 3.2 If $F$ is a monotone quasi-periodically forced map then so is $F^{n}$, and hence in particular

$$
g^{(n)}(\theta, y) \leq g^{(n)}\left(\theta, y^{\prime}\right)
$$

for all $y \leq y^{\prime}$, all $\theta \in \mathbb{T}^{1}$ and all $n \in \mathbb{N}$.

Proof This follows by straightforward induction using the previous lemma with $m=1$. The result holds for $n=1$ by definition. Suppose it holds for some $n>1$, so that $g^{(n)}(\theta, y) \leq g^{(n)}\left(\theta, y^{\prime}\right)$ for all $y \leq y^{\prime}$ and all $\theta \in \mathbb{T}^{1}$. Then if $y \leq y^{\prime}$, the inductive hypothesis implies that $g\left(f^{n}(\theta), g^{(n)}(\theta, y)\right)$ $\leq g\left(f^{n}(\theta), g^{(n)}\left(\theta, y^{\prime}\right)\right)$. Hence by the previous lemma $g^{(n+1)}(\theta, y) \leq g^{(n+1)}\left(\theta, y^{\prime}\right)$, as required.

Definition 3.1 Let $\xi^{(n)}: \mathbb{T}^{1} \times \mathbb{R} \rightarrow \mathbb{R}$ be the function

$$
\xi^{(n)}(\theta, y)=g^{(n)}(\theta, y)-y
$$


The next lemma is also trivial, but when combined with Corollary 3.2, yields a key estimate which in turn implies that $\xi^{(n)}$ is in a certain sense sub-additive. This latter property underlies the whole proof of Theorem 1.2.

Lemma 3.3 If $F$ is a monotone quasi-periodically forced circle map then $\xi^{(n)}$ is periodic in $y$ for any $\theta \in \mathbb{T}^{1}$, that is

$$
\xi^{(n)}(\theta, y+k) \quad=\quad \xi^{(n)}(\theta, y)
$$

for all $(\theta, y) \in \mathbb{T}^{1} \times \mathbb{R}$, all $n \in \mathbb{N}$ and all $n \in \mathbb{Z}$.

Proof This follows by straightforward induction. It clearly suffices to just prove the result for $k=1$. Since $g(\theta, y+1)=g(\theta, y)+1$, we have by definition that $\xi^{(1)}(\theta, y+1)=g(\theta, y+1)-y-1=g(\theta, y)$ $+1-y-1=\xi^{(1)}(\theta, y)$. Now suppose that for a given $n$, we have $\xi^{(n)}(\theta, y+1)=\xi^{(n)}(\theta, y)$ for all $(\theta, y) \in$ $\mathbb{T}^{1} \times \mathbb{R}$. Then $\xi^{(n+1)}(\theta, y+1)=g^{(n+1)}(\theta, y+1)-y-1=g\left(f^{n}(\theta), g^{(n)}(\theta, y+1)\right)-y-1$ by Lemma 3.1. By definition and the inductive hypothesis, $g^{(n)}(\theta, y+1)=\xi^{(n)}(\theta, y+1)+y+1=\xi^{(n)}(\theta, y)+y+1=g^{(n)}(\theta, y)$ +1 , and hence $\xi^{(n+1)}(\theta, y+1)=g\left(f^{n}(\theta), g^{(n)}(\theta, y)+1\right)-y-1=g\left(f^{n}(\theta), g^{(n)}(\theta, y)\right)+1-y-1=\xi^{(n+1)}(\theta, y)$, as required.

Corollary 3.4 [Herman, 1979] If $F$ is a monotone quasi-periodically forced circle map then for all $\theta \in \mathbb{T}^{1}, y, y^{\prime} \in \mathbb{R}$ and $n \in \mathbb{N}$

$$
\left|\xi^{(n)}(\theta, y)-\xi^{(n)}\left(\theta, y^{\prime}\right)\right| \quad \leq \quad 1
$$

Proof Since $\xi^{(n)}\left(\theta, y^{\prime}\right)=\xi^{(n)}\left(\theta, y^{\prime}+k\right)$ for all $n \in \mathbb{Z}$, and the result is trivial if $y=y^{\prime}$, we may assume without loss of generality that $y<y^{\prime}<y+1$. Now $\xi^{(n)}(\theta, y)=g^{(n)}(\theta, y)-y \leq g^{(n)}(\theta, y)-y$ and since $-y$ $<-y^{\prime}+1$ we have $\xi^{(n)}(\theta, y) \leq g^{(n)}\left(\theta, y^{\prime}\right)-y^{\prime}+1=\xi^{(n)}\left(\theta, y^{\prime}\right)+1$. Hence

$$
\xi^{(n)}(\theta, y)-\xi^{(n)}\left(\theta, y^{\prime}\right) \quad \leq \quad 1
$$

On the other hand, $\xi^{(n)}(\theta, y)=\xi^{(n)}(\theta, y+1)=g^{(n)}(\theta, y+1)-y-1 \geq g^{(n)}(\theta, y)-y$ - 1 . Since $-y>-y^{\prime}$, this yields $\xi^{(n)}(\theta, y) \geq g^{(n)}\left(\theta, y^{\prime}\right)-y^{\prime}-1=\xi^{(n)}\left(\theta, y^{\prime}\right)-1$. Hence

$$
\xi^{(n)}(\theta, y)-\xi^{(n)}\left(\theta, y^{\prime}\right) \quad \geq \quad-1
$$

Combining (3.2) and (3.3) gives the required result.

Applying this to $\xi^{(n+m)}$ and unravelling the definitions leads to the following sub-additive property for $\xi^{(n)}$ :

Corollary 3.5 If $F$ is a monotone quasi-periodically forced circle map then for all $\theta \in \mathbb{T}^{1}, y, y^{\prime} \in \mathbb{R}$ and $n, m \in \mathbb{N}$ we have

$$
\left|\xi^{(n+m)}(\theta, y)-\xi^{(m)}\left(f^{n}(\theta), y^{\prime}\right)-\xi^{(n)}(\theta, y)\right| \leq 1
$$

Proof By Lemma 3.1, we have 


$$
\begin{array}{rlrl}
\xi^{(n+m)}(\theta, y) & = & g^{(n+m)}(\theta, y)-y \\
& = & g^{(m)}\left(f^{n}(\theta), g^{(n)}(\theta, y)\right)-y \\
& = & g^{(m)}\left(f^{n}(\theta), g^{(n)}(\theta, y)\right)-g^{(n)}(\theta, y)+g^{(n)}(\theta, y)-y \\
& =\quad \xi^{(m)}\left(f^{n}(\theta), g^{(n)}(\theta, y)\right)+\xi^{(n)}(\theta, y)
\end{array}
$$

But by (3.1) we have $\left|\xi^{(m)}\left(f^{n}(\theta), g^{(n)}(\theta, y)\right)-\xi^{(m)}\left(f^{n}(\theta), y^{\prime}\right)\right| \leq 1$ for any $y^{\prime} \in \mathbb{R}$, immediately giving the required bound (3.4).

\subsection{ITERATION OF CURVES}

Definition 3.2 Given any function $\psi: \mathbb{T}^{1} \rightarrow \mathbb{R}$, define $\xi_{n}: \mathbb{T}^{1} \rightarrow \mathbb{R}$ by

$$
\xi_{n}(\theta) \quad=\quad \xi^{(n)}(\theta, \psi(\theta))
$$

Observe that this is the amount of vertical distance that the point $(\theta, \psi(\theta))$ moves in $n$ iterations. If the rotation number exists, then we should have $\frac{1}{n} \xi_{n}(\theta) \rightarrow \rho$ for all $\theta \in \mathbb{T}^{1}$. We first show that in an appropriate sense $\left\{\xi_{n}\right\}$ is both a sub- and a super-additive sequence of functions. More precisely.

Lemma 3.6 If $F$ is a monotone quasi-periodically forced circle map then for all $\theta \in \mathbb{T}^{1}$ and $n, m \in \mathbb{N}$ we have

$$
\left|\xi_{n+m}(\theta)-\xi_{m}\left(f^{n}(\theta)\right)-\xi_{n}(\theta, y)\right| \leq 1
$$

Proof Let $y^{\prime}(\theta)=\psi\left(f^{n}(\theta)\right)$, then $\xi_{m}\left(f^{n}(\theta)\right)=\xi^{(m)}\left(f^{n}(\theta), y^{\prime}(\theta)\right)$. Thus by (3.4) we have $\mid \xi_{n+m}(\theta)$ $\xi_{m}\left(f^{n}(\theta)\right)-\xi_{n}(\theta, y)|=| \xi^{(n+m)}(\theta, \psi(\theta))-\xi^{(m)}\left(f^{n}(\theta), y^{\prime}(\theta)\right)-\xi^{(n)}(\theta, \psi(\theta)) \mid \leq 1$, as required.

Definition 3.3 With $\xi_{n}$ as above, if $\psi$ and $F$ are measurable define

$$
\rho_{n} \quad=\quad \int \xi_{n} d \theta
$$

and

$$
\begin{array}{lll}
\rho_{n}^{+} & = & \rho_{n}+1 \\
\rho_{n}^{-} & = & \rho_{n}-1
\end{array}
$$

Corollary 3.7 If $F$ is a monotone measurable quasi-periodically forced circle map then $\rho_{n}^{+}$is a sub-additive sequence and $\rho_{n}^{-}$a super-additive one, that is

$$
\begin{array}{lll}
\rho_{n+m}^{+} & \leq & \rho_{m}^{+}+\rho_{n}^{+} \\
\rho_{n+m}^{-} & \geq & \rho_{m}^{-}+\rho_{n}^{-}
\end{array}
$$

Proof Since Lebesque measure is invariant under $f$, we trivially have 


$$
\int \varphi \circ f^{-n} d \theta \quad=\quad \int \varphi d \theta
$$

for any integrable $\varphi: \mathbb{T}^{1} \rightarrow \mathbb{R}$ and any $n \in \mathbb{Z}$. Hence by (3.5) we have

$$
-1 \leq \rho_{n+m}-\rho_{m}-\rho_{n} \leq 1
$$

and hence by definition $\rho_{n+m}^{+}-1-\rho_{m}^{+}+1-\rho_{n}^{+}+1 \leq 1$, so that $\rho_{n+m}^{+} \leq \rho_{m}^{+}+\rho_{n}^{+}$as claimed. Similarly $\rho_{n+m}^{-}+1-\rho_{m}^{-}-1-\rho_{n}^{-}-1 \geq-1$, so that $\rho_{n+m}^{-} \geq \rho_{m}^{-}+\rho_{n}^{-}$.

Now recall that by the sub-additive lemma (e.g. [Katok and Hasselblatt, 1995]) if $\left\{a_{n}\right\}$ is a sub-additive sequence, then $\frac{1}{n} a_{n}$ converges to a limit $a$ (with possibly $a=-\infty$ if $\frac{1}{n} a_{n}$ is not bounded below). Furthermore $a \leq \frac{1}{n} a_{n}$ for any $n \in \mathbb{N}$. Hence we immediately have:

Lemma 3.8 If $F$ is a monotone quasi-periodically forced circle map then $\rho_{n}^{+}$and $\rho_{n}^{-}$converge to a common limit $\hat{\rho}$, and

$$
\hat{\rho}-\frac{1}{n} \quad \leq \quad \frac{1}{n} \rho_{n} \leq \hat{\rho}+\frac{1}{n}
$$

for all $n \in \mathbb{N}$.

Proof By repeated application of Corollary 3.7 we have $n \rho_{1}^{-} \leq \rho_{n}^{-}$. and $\rho_{n}^{+} \leq n \rho_{1}^{+}$. By definition $\rho_{n}^{-}$ $<\rho_{n}^{+}$, so that $\rho_{1}^{-}<\frac{1}{n} \rho_{n}^{+}$and $\frac{1}{n} \rho_{n}^{-}$. $<\rho_{1}^{+}$. Thus $\frac{1}{n} \rho_{n}^{+}$is bounded below and $\frac{1}{n} \rho_{n}^{-}$is bounded above. Hence both converge to finite limits, say $\hat{\rho}^{+}$and $\hat{\rho}^{-}$respectively. Since $\hat{\rho}_{n}^{-}=\rho_{n}^{+}-2$, we have $\hat{\rho}^{-}=$ $\hat{\rho}^{+}=\hat{\rho}$. Furthermore, by the sub-additive lemma, $\hat{\rho} \leq \frac{1}{n} \rho_{n}^{+}=\frac{1}{n}\left(\rho_{n}+1\right)$. Hence $\hat{\rho}-\frac{1}{n} \leq \frac{1}{n} \rho_{n}$. Similarly $\hat{\rho}$ $\geq \frac{1}{n} \rho_{n}^{-}=\frac{1}{n}\left(\rho_{n}-1\right)$ so that $\hat{\rho}+\frac{1}{n} \geq \frac{1}{n} \rho_{n}$.

\subsection{THE Rotation NUMBER}

Observe that (3.7)is exactly the same bound as (1.9), except that it is in terms of $\hat{\rho}$ rather than $\rho$. All that remains to do to prove Theorem 1.2 is therefore to show that $\hat{\rho}$ is in fact the rotation number. This can readily be deduced from the uniform convergence of $\frac{1}{n}\left(g^{(n)}(\theta, y)-y\right)$ (Theorem 1.1). However, Herman's proof of this result is not easily accessible at an elementary level. For the benefit of the reader, we therefore present a much simpler argument based on the sub-additive bound (3.4). It turns out that this gives a simultaneous proof of both Theorem 1.1 and Theorem 1.2. First recall (e.g. see [Katok and Hasselblatt, 1995] or [Arnold, 1998]):

Definition 3.4 Suppose that $T: X \rightarrow X$ is a measurable map on a metrizable space $X$. We say that a sequence of functions $\varphi_{n}: X \rightarrow \mathbb{R}$ is sub-additive if $\varphi_{n+m}(x) \leq \varphi_{n}(x)+\varphi_{m}\left(T^{n}(x)\right)$ for all $x \in$ $X$.

Theorem 3.1 (Sub-Additive Ergodic Theorem, e.g. see [Katok and Hasselblatt, 1995] or [Arnold, 1998]) Let $T: X \rightarrow X$ be a measurable map, $\mu$ a $T$-invariant measure and $\left\{\varphi_{n}\right\}$ an integrable sub-additive sequence. Then the limit

$$
\lim _{n \rightarrow \infty} \frac{1}{n} \varphi_{n}(x) \quad=\quad \bar{\varphi}(x)
$$

exists for $\mu$-almost every $x$. Furthermore $\bar{\varphi}$ is $T$-invariant, integrable and $\frac{1}{n} \bar{\varphi}_{n} \rightarrow \bar{\varphi}$ in $\mathcal{L}^{1}$. 
Note that that the $T$-invariance of $\bar{\varphi}$ means that if $\mu$ is ergodic then $\bar{\varphi}$ is constant $\mu$-almost everywhere. Observe that (3.5) means that $\varphi_{n}=\psi_{n}+1$ is sub-additive for any initial $\psi$, and hence for instance taking $\psi(\theta)=y_{0}$ we see that $\frac{1}{n}\left(g^{(n)}\left(\theta, y_{0}\right)-y_{0}\right)$ converges to a constant for Lebesque almost all $\theta$. If, however, we want to show convergence for all $(\theta, y) \in \mathbb{T}^{1} \times \mathbb{R}$, we need some kind of uniformity of convergence in Theorem 3.1. This is not unreasonable to hope for given that $f$ is uniquely ergodic and:

Theorem 3.2 (see e.g. [Katok and Hasselblatt, 1995]) Suppose that $T: X \rightarrow X$ is a uniquely ergodic measurable map on a compact metrizable space $X$, and $\varphi: X \rightarrow \mathbb{R}$ is a continuous function. Then the time average

$$
\lim _{n \rightarrow \infty} \frac{1}{n} \sum_{i=0}^{n-1} \varphi\left(T^{i}(x)\right)
$$

converges uniformly to $\int \varphi d \mu$ for all $x$ (where $\mu$ is the unique invariant measure).

Unfortunately, it is turns out that the generalization of this theorem to the sub-additive case is false [Derriennic and Krengel, 1981]. In other words one does not get uniform convergence in (3.8) even for uniquely ergodic systems. However, observe that in our case (3.5) implies that $\psi_{n}+1$ is sub-additive and $\psi_{n}-1$ is super-additive (i.e. $-\psi_{n}+1$ is also sub-additive). It is thus sufficient to obtain uniformity from above for the convergence of $\frac{1}{n}\left(\psi_{n}+1\right)$ and from below for that of $\frac{1}{n}\left(\psi_{n}-1\right)$. This is provided by the following theorem, which is essentially proved in [Stark, 1997], though not explicitly stated there. Since the proof is an elementary estimate, it is given here for the benefit of the reader. The theorem can also be easily deduced from the results of Slomczynski [1995][1997]. A subset of these results was independently derived in [Sturman and Stark, 2000], where they are used to characterize certain properties of invariant sets for quasi-periodically forced systems.

Theorem 3.3 (Semi-Uniform Sub-Additive Ergodic Theorem) Suppose that $T: X \rightarrow X$ is a uniquely ergodic measurable map on a compact metrizable space $X$, and $\left\{\varphi_{n}\right\}$ a sub-additive sequence of continuous functions $\varphi_{n}: X \rightarrow \mathbb{R}$. Then given $\varepsilon>0$, there exists an $N \in \mathbb{N}$ such that for all $n \geq N$ we have

$$
\frac{1}{n} \varphi_{n}(x) \quad \leq \quad \bar{\varphi}+\varepsilon
$$

for all $x \in X$, where $\bar{\varphi}$ is the limit (3.8), which is necessarily constant almost everywhere with respect to the unique $T$-invariant measure.

Proof Denote $\bar{\varphi}_{n}=\frac{1}{n} \int \varphi_{n} d \mu$ and observe that $\bar{\varphi}_{n} \rightarrow \bar{\varphi}$ as $n \rightarrow \infty$. Hence given $\varepsilon>0$, choose $n$ such that $\bar{\varphi}_{n} \leq \bar{\varphi}+\varepsilon$. Let $K$ be the supremum of $\varphi_{1}, \ldots, \varphi_{n}$ over $X$ (using the compactness of $X$ ). Then for any $k \in \mathbb{N}$, and any $0 \leq j<n$, by repeatedly applying the sub-additive condition we obtain

$$
\varphi_{k n}(\theta) \leq \varphi_{j}(\theta)+\varphi_{n-j}\left(f^{(k-1) n+j}(\theta)\right)+\sum_{i=0}^{k-2} \varphi_{n}\left(f^{i n+j}(\theta)\right)
$$

Summing over $j=0, \ldots, n$-1 we obtain 


$$
\begin{aligned}
\varphi_{k n}(\theta) & \leq \frac{1}{n} \sum_{j=0}^{n-1} \sum_{i=0}^{k-2} \varphi_{n}\left(f^{i n+j}(\theta)\right)+\frac{1}{n} \sum_{j=0}^{n-1}\left[\varphi_{j}(\theta)+\varphi_{n-j}\left(f^{(k-1) n+j}(\theta)\right)\right] \\
& \leq \sum_{j=0}^{(k-1) n-1} \frac{1}{n} \varphi_{n}\left(f^{j}(\theta)\right)+2 K
\end{aligned}
$$

Since $\varphi_{n}$ is continuous and $T$ is uniquely ergodic, by Theorem 3.2 there exists $N$ such that for all $m \geq N$ we have

$$
\frac{1}{m} \sum_{j=0}^{m-1} \frac{1}{n} \varphi_{n}\left(f^{j}(\theta)\right) \quad \leq \quad \int \frac{1}{n} \varphi_{n} d \theta+\varepsilon
$$

Hence for all $k$ such that $n(k-1) \geq N$ we have

$$
\begin{aligned}
\varphi_{k n}(\theta) \quad & \leq \quad(k-1) n\left(\bar{\varphi}_{n}+\varepsilon\right)+2 K \\
& \leq \quad(k-1) n \bar{\varphi}+2(k-1) n \varepsilon+2 K
\end{aligned}
$$

Finally, for any $m \geq M+2 n$, write $m=k n+j$, with $0 \leq j<n$. Thus $(k-1) n=m-j-n>M$. By sub-additivity, $\varphi_{m}(\theta) \leq \varphi_{k n}(\theta)+\varphi_{j}\left(f^{k n}(\theta)\right)$, and hence

$$
\varphi_{m}(\theta) \quad \leq \quad(k-1) n \bar{\varphi}+2(k-1) n \varepsilon+3 K
$$

Now, $(k-1) n<m$, and thus

$$
\frac{1}{m} \varphi_{m}(\theta) \quad \leq \quad \bar{\varphi}+2 \varepsilon+\frac{3}{m} K
$$

Hence for $m \geq \max \left\{M+2 n, \frac{K}{3 \varepsilon}\right\}$ we obtain

$$
\frac{1}{m} \varphi_{m}(\theta) \quad \leq \quad \bar{\varphi}+3 \varepsilon
$$

as required.

As an immediate consequence we obtain proofs of both Theorem 1.1 and Theorem 1.2. Thus pick an arbitrary $y_{0}$. By (3.4), both $\xi^{(n)}\left(\theta, y_{0}\right)+1$ and $-\xi^{(n)}\left(\theta, y_{0}\right)+1$ are sub-additive sequences of functions (and are continuous if $F$ is). By Lemma 3.8, we have

$$
\begin{array}{lll}
\lim _{n \rightarrow \infty} \frac{1}{n} \int\left(\xi^{(n)}\left(\theta, y_{0}\right)+1\right) d \theta & = & \hat{\rho} \\
\lim _{n \rightarrow \infty} \frac{1}{n} \int\left(-\xi^{(n)}\left(\theta, y_{0}\right)+1\right) d \theta & = & -\hat{\rho}
\end{array}
$$

Hence by Theorem 3.3, given $\varepsilon>0$, there exists $N$ such that for all $n \geq N$ and $\theta \in \mathbb{T}^{1}$ we have

$$
\begin{array}{lll}
\frac{1}{n}\left(\xi^{(n)}\left(\theta, y_{0}\right)+1\right) & \leq & \hat{\rho}+\varepsilon \\
\frac{1}{n}\left(-\xi^{(n)}\left(\theta, y_{0}\right)+1\right) & \leq & -\hat{\rho}+\varepsilon
\end{array}
$$

Hence 


$$
\hat{\rho}-\varepsilon-\frac{1}{n} \quad \leq \quad \frac{1}{n} \xi^{(n)}\left(\theta, y_{0}\right) \leq \hat{\rho}+\varepsilon-\frac{1}{n}
$$

$n \geq N$ and $\theta \in \mathbb{T}^{1}$. Thus by Corollary 3.4, we have for all $n \geq N$ and all $(\theta, y) \in \mathbb{T}^{1} \times \mathbb{R}$ that

$$
\hat{\rho}-\varepsilon \quad \leq \quad \frac{1}{n} \xi^{(n)}(\theta, y) \quad \leq \quad \hat{\rho}+\varepsilon
$$

This shows both that $\hat{\rho}=\rho$, completing the proof of Theorem 1.2 and that $\frac{1}{n}\left(g^{(n)}(\theta, y)-y\right)$ converges uniformly to $\hat{\rho}$, thus giving a proof of Theorem 1.1. Note also that we have shown that $\hat{\rho}$ is independent of the choice of initial curve $\psi$, which can also be done directly using (3.1).

\section{BOUNDED AND UNBOUNDED ORBITS}

In this section we give a proof of Theorem 1.3. To simplify the notation, it is convenient to define:

Definition 4.1 For a fixed $\rho$, define $\zeta^{(n)}: \mathbb{T}^{1} \times \mathbb{R} \rightarrow \mathbb{R}$ to be the function

$$
\zeta^{(n)}(\theta, y)=g^{(n)}(\theta, y)-y-\rho n
$$

A very similar calculation to Corollary 3.4 gives

Lemma 4.1 With $\zeta^{(n)}$ defined as above, we have

$$
\begin{array}{rlrl}
\zeta^{(m+n)}\left(\theta_{0}, y_{0}\right) & = & \zeta^{(m)}\left(\theta_{n}, y_{n}\right)+y_{n}-y_{0}-\rho n \\
& =\quad \zeta^{(m)}\left(\theta_{n}, y_{n}\right)+\zeta^{(n)}\left(\theta_{0}, y_{0}\right)
\end{array}
$$

for all $n, m \in \mathbb{N}$ and $\left(\theta_{0}, y_{0}\right) \in \mathbb{T}^{1} \times \mathbb{R}$.

Proof By Lemma 3.1, we have

$$
\begin{aligned}
\zeta^{(n+m)}\left(\theta_{0}, y_{0}\right) & = & g^{(n+m)}\left(\theta_{0}, y_{0}\right)-y_{0}-\rho(m+n) \\
& = & g^{(m)}\left(f^{n}\left(\theta_{0}\right), g^{(n)}\left(\theta_{0}, y_{0}\right)\right)-y_{0}-\rho(m+n) \\
& = & \zeta^{(m)}\left(\theta_{n}, y_{n}\right)+y_{n}+\rho m-y_{0}-\rho(m+n)
\end{aligned}
$$

Furthermore, by definition $y_{n}=g^{(n)}\left(\theta_{0}, y_{0}\right)=\zeta^{(n)}\left(\theta_{0}, y_{0}\right)+y_{0}+\rho n$, so that $\zeta^{(m)}\left(\theta_{n}, y_{n}\right)+y_{n}-y_{0}-\rho n=$ $\zeta^{(m)}\left(\theta_{n}, y_{n}\right)+\zeta^{(n)}\left(\theta_{0}, y_{0}\right)$, as required.

The next two lemmas establish the basic dichotomy between the $\rho$-bounded and the $\rho$ unbounded situations. More precisely, they show that the sets of $\rho$-bounded, $\rho$-unbounded above and $\rho$-unbounded below orbits are each either empty or residual.

Lemma 4.2 If there exists an orbit that is $\rho$-unbounded above then there exists a residual subset $U \subset \mathbb{T}^{1}$ such that all $(\theta, y) \in U \times \mathbb{R}$ are $\rho$-unbounded above. Similarly if there is a $\rho$ unbounded below orbit then there is a residual subset on which orbits are $\rho$-unbounded below. 
Proof Suppose that for some $\left(\theta_{0}, y_{0}\right)$ we have

$$
\limsup _{n \rightarrow \infty} \zeta^{(n)}\left(\theta_{0}, y_{0}\right) \quad=\quad \infty
$$

Choose $M \in \mathbb{N}$. Given any $n \in \mathbb{N}$, we claim that there exists an $m(n)$ such that $\zeta^{m(n)}\left(\theta_{n}, y_{n}\right) \geq M+2$. This is because if not, so that $\zeta^{(m)}\left(\theta_{n}, y_{n}\right)<M+2$ for all $m \in \mathbb{N}$, then by Lemma 4.1, $\zeta^{(n+m)}\left(\theta_{0}, y_{0}\right)<$ $M+2+y_{n}-y_{0}-\rho n$, and hence $\zeta^{(k)}\left(\theta_{0}, y_{0}\right) \leq K+M+2+y_{n}-y_{0}-\rho n$, where $K=\max \left\{y_{0}, \ldots, y_{n-1}\right\}$. This contradicts the assumption that the orbit of $\left(\theta_{0}, y_{0}\right)$ is $\rho$-unbounded above.

By the continuity of $g$, we can choose open intervals $U_{n}^{M}$ such that $\zeta^{m(n)}\left(\theta, y_{n}\right) \geq M+1$ for all $\theta \in$ $U_{n}^{M}$. Then by Corollary 3.4, $\zeta^{m(n)}(\theta, y) \geq M$ for all $(\theta, y) \in U_{n}^{M} \times \mathbb{R}$. Define

$$
U^{M} \quad=\quad \bigcup_{n \in \mathbb{N}} U_{n}^{M}
$$

Since $\left\{\theta_{n}\right\}$ is dense in $\mathbb{T}^{1}$, this is a dense open set, and clearly if $\theta \in U^{M}$, there exists some $m \in \mathbb{N}$ for which $\zeta^{(m)}(\theta, y) \geq M$ for all $y \in \mathbb{R}$. Now define the residual set $U$ by

$$
U \quad=\bigcap_{M \in \mathbb{N}} U^{M}
$$

If $\theta \in U$, then $\theta \in U^{M}$ for all $M \in \mathbb{N}$. Hence for each $M \in \mathbb{N}$ there exists an $m$ such that $\zeta^{(m)}(\theta, y) \geq M$ for all $y \in \mathbb{R}$. Thus

$$
\limsup _{n \rightarrow \infty} \zeta^{(n)}(\theta, y) \quad=\quad \infty
$$

for all $(\theta, y) \in U \times \mathbb{R}$.

Lemma 4.3 If there exists an orbit that is $\rho$-bounded then there exists a residual subset $U \subset$ $\mathbb{T}^{1}$ such that the orbit of any $(\theta, y) \in U \times \mathbb{R}$ is $\rho$-bounded.

Proof Suppose that for some $\left(\theta_{0}, y_{0}\right) \in \mathbb{T}^{1} \times \mathbb{R}$ we have

$$
\left|\zeta^{(n)}\left(\theta_{0}, y_{0}\right)\right| \leq \quad C
$$

for all $n \in \mathbb{N}$ for some constant $C>0$. Then by Lemma 4.1, $\zeta^{(m)}\left(\theta_{n}, y_{n}\right)=\zeta^{(m+n)}\left(\theta_{0}, y_{0}\right)-\zeta^{(n)}\left(\theta_{0}, y_{0}\right)$ and hence $\left|\zeta^{(m)}\left(\theta_{n}, y_{n}\right)\right| \leq 2 C$, for all $m \in \mathbb{N}$. Hence, as in Lemma 4.2 above, we can choose open intervals $U_{m n}$ such that $\left|\zeta^{(m)}\left(\theta, y_{n}\right)\right| \leq 2 C+1$ for all $\theta \in U_{m n}$. Then by Corollary 3.4, $\left|\zeta^{(m)}(\theta, y)\right| \leq 2 C+2$ for all $(\theta, y) \in U_{m n} \times \mathbb{R}$. Define

$$
U_{m} \quad=\quad \bigcup_{n \in \mathbb{N}} U_{m n}
$$

Since $\left\{\theta_{m}\right\}$ is dense in $\mathbb{T}^{1}$, this is a dense open set, and $\left|\zeta^{(m)}(\theta, y)\right| \leq 2 C+2$ for all $(\theta, y) \in U_{m} \times \mathbb{R}$. Now let

$$
U \quad=\quad \bigcap_{m \in \mathbb{N}} U_{m}
$$

This is a residual set, and if $\theta \in U$, then $\theta \in U_{m}$ for all $m \in \mathbb{N}$. Hence $\left|\zeta^{(m)}(\theta, y)\right| \leq 2 C+2$ for all $m \in \mathbb{N}$ for all $(\theta, y) \in U \times \mathbb{R}$. In other words the orbit of $(\theta, y)$ is $\rho$-bounded. 
The two lemmas together have the immediate corollary

Corollary 4.4 If there exists a $\rho$-bounded orbit then all orbits are $\rho$-bounded.

Proof Suppose not, so that there exists a $\rho$-bounded orbits, but not all orbits are $\rho$-bounded. Thus there exists either a $\rho$-unbounded above or a $\rho$-unbounded below orbit. Without loss of generality, assume the former. Then the above two lemmas show that there exist residual sets $U_{0}, U_{1} \subset \mathbb{T}^{1}$ such that if $(\theta, y) \in U_{0} \times \mathbb{R}$ then the orbit of $(\theta, y)$ is $\rho$-bounded, whilst if $(\theta, y) \in$ $U_{1} \times \mathbb{R}$ then the orbit of $(\theta, y)$ is $\rho$-unbounded. The intersection of $U_{0}$ and $U_{1}$ is residual, and hence in particular non-empty. But if $\theta$ lies in the intersection then the orbit of $(\theta, y)$, for any $y \in \mathbb{R}$, is both $\rho$-bounded and $\rho$-unbounded above, which is clearly not possible.

To prove Theorem 1.3, it remains to show that if there exists a $\rho$-unbounded above orbit, then there exists one which is $\rho$-unbounded below, and vice versa. This follows from the following characterization of the rotation number, which is the closest generalization of (1.6) that we have been able to derive in the quasi-periodically forced case.

Lemma 4.5 Suppose that for all $\theta \in \mathbb{T}^{1}$ there exists a $y(\theta) \in \mathbb{R}$ and a $m(\theta) \in \mathbb{N}$ such that $\zeta^{(m(\theta))}(\theta, y(\theta))>1$. Then $\rho(F)>\rho$. Similarly if $\zeta^{(m(\theta))}(\theta, y(\theta))<-1$, then $\rho(F)<\rho$.

Proof Let $\varepsilon(\theta)=\frac{1}{2}\left(\zeta^{(m(\theta)}(\theta, y(\theta))\right.$ - 1). By continuity, for each $\theta \in \mathbb{T}^{1}$ choose an open interval $U(\theta)$ $\subset \mathbb{T}^{1}$ such that $\zeta^{(m(\theta)}\left(\theta^{\prime}, y(\theta)\right)>1+\varepsilon(\theta)$ for all $\theta^{\prime} \in U(\theta)$. The $U(\theta)$ form an open cover of $\mathbb{T}^{1}$, so since $\mathbb{T}^{1}$ is compact we may choose a finite sub-cover, say $U\left(\Theta_{1}\right), \ldots, U\left(\Theta_{k}\right)$. For convenience, write $U_{i}=U\left(\Theta_{i}\right)$ and define $\varepsilon=\min \left\{\varepsilon\left(\Theta_{1}\right), \ldots, \varepsilon\left(\Theta_{k}\right)\right\}$ and $M=\max \left\{m\left(\Theta_{1}\right), \ldots, m\left(\Theta_{k}\right)\right\}$.

Now, given any $\left(\theta_{0}, y_{0}\right) \in \mathbb{T}^{1} \times \mathbb{R}$ inductively define sequences $\left\{n_{i}\right\},\left\{k_{i}\right\}$ and $\left\{m_{i}\right\}$ by $n_{0}=0, k_{i}$ is such that $\theta_{n_{i}} \in U_{k_{i}}, m_{i}=m_{k_{i}}\left(\Theta_{k_{i}}\right)$ and $n_{k+1}=n_{i}+m_{i}$. Since $\theta_{n_{i}} \in U_{k_{i}}$ we have $\zeta^{\left(m_{j}\right.}\left(\theta_{n_{i}} y(\theta)\right)>1+\varepsilon$ and hence by Corollary 3.4, $\zeta^{\left.m_{j}\right)}\left(\theta_{n_{i}} y_{n_{i}}\right)>\varepsilon$. Now, by Lemma 4.1, $\zeta^{\left(n_{i+1}\right)}\left(\theta_{0}, y_{0}\right)=\zeta^{\left(m_{+}+n_{j}\right)}\left(\theta_{0}, y_{0}\right)=$ $\zeta^{m_{j} j}\left(\theta_{n_{i}}, y_{n_{i}}\right)+\zeta^{\left(n_{j}\right.}\left(\theta_{0}, y_{0}\right)$. Hence $\zeta^{\left(n_{i_{1+1}}\right)}\left(\theta_{0}, y_{0}\right) \geq \zeta^{\left(n_{j}\right)}\left(\theta_{0}, y_{0}\right)+\varepsilon$. By induction $\zeta^{\left(n_{j}\right)}\left(\theta_{0}, y_{0}\right) \geq \varepsilon i$ for all $i \in \mathbb{N}$. On the other hand $m_{i} \leq M$ for all $i \in \mathbb{N}$, and hence $n_{i} \leq M i$. Thus

$$
\begin{aligned}
\limsup _{n \rightarrow \infty} \frac{1}{n} \zeta^{(n)}\left(\theta_{0}, y_{0}\right) & \geq \limsup _{i \rightarrow \infty} \frac{1}{n_{i}} \zeta^{\left(n_{j}\right)}\left(\theta_{0}, y_{0}\right) \\
& \geq \frac{\varepsilon}{M}
\end{aligned}
$$

But by Theorem 1.1 we have for any $\left(\theta_{0}, y_{0}\right) \in \mathbb{T}^{1} \times \mathbb{R}$ that

$$
\begin{aligned}
\rho(F) \quad & =\limsup _{n \rightarrow \infty} \frac{1}{n}\left(g^{(n)}\left(\theta_{0}, y_{0}\right)-y_{0}\right) \\
& =\quad \limsup _{n \rightarrow \infty} \frac{1}{n}\left(\zeta^{(n)}\left(\theta_{0}, y_{0}\right)+\rho n\right) \\
& \geq \quad \frac{\varepsilon}{M}+\rho
\end{aligned}
$$

Hence $\rho(F)>\rho$, as claimed.

We immediately get:

Corollary 4.6 If the rotation number of $F$ is $\rho$, then not all orbits can be $\rho$-unbounded above (or below). 
Corollary 4.7 If the rotation number of $F$ is $\rho$, and there exists a $\rho$-unbounded above orbit, then there exists one which is $\rho$-unbounded below, and vice versa.

Proof If there exists a $\rho$-unbounded above orbit then by Lemma 4.3, there cannot be any $\rho$ bounded orbits. By Corollary 4.6 there must be an orbit that is not $\rho$-unbounded above. Since it cannot be $\rho$-bounded, it must be $\rho$-unbounded below, as required.

We thus see that if there is an $\rho$-unbounded orbit, then (by Lemma 4.2) there are residual sets of orbits that are respectively $\rho$-unbounded above and $\rho$-unbounded below. Taking the intersection, we obtain a residual set of orbits that are $\rho$-unbounded both above and below, thereby completing the proof of Theorem 1.3.

\section{Min-Max ChaRACterizations OF THE Rotation Number}

In this final section, we show that Lemma 4.5 leads to a characterization of the rotation number which can be used for numerical estimation. Like Theorem 1.2 this provides rigorous bounds on $\rho$. Given a arbitrary function $\psi: \mathbb{T}^{1} \rightarrow \mathbb{R}$ define

$$
\begin{array}{lll}
\alpha_{n}^{-}(\theta) & = & \max _{1 \leq m \leq n} \frac{1}{m}\left(g^{(m)}(\theta, \psi(\theta))-\psi(\theta)-1\right) \\
\alpha_{n}^{+}(\theta) & = & \min _{1 \leq m \leq n} \frac{1}{m}\left(g^{(m)}(\theta, \psi(\theta))-\psi(\theta)+1\right)
\end{array}
$$

Observe that for each $m$ the function $g^{(m)}(\theta, 0)$ is bounded below since $g$ is continuous and $\mathbb{T}^{1}$ is compact. Hence by Corollary 3.4, so is $g^{(m)}(\theta, \psi(\theta))-\psi(\theta)$, and thus $\alpha_{n}(\theta)$ is also bounded below for each fixed $n$. Similarly $\alpha_{n}^{+}(\theta)$ is bounded above. Define

$$
\begin{array}{lll}
\dot{\alpha_{n}} & = & \inf _{\theta \in \mathbb{T}^{1}} \\
\alpha_{n}(\theta) \\
\alpha_{n}^{+} & = & \sup _{\theta \in \mathbb{T}^{1}} \alpha_{n}^{+}(\theta)
\end{array}
$$

As a straightforward consequence of at Lemma 4.5, we obtain

Lemma 5.1 If $F$ has rotation number $\rho$, then

$$
\overline{\alpha_{n}} \leq \rho \leq \alpha_{n}^{+}
$$

Proof For each $\theta \in \mathbb{T}^{1}$ there exists an $m(\theta) \in \mathbb{N}$ such that

$$
\begin{aligned}
\frac{1}{m(\theta)}\left(g^{(m(\theta))}(\theta, \psi(\theta))-\psi(\theta)-1\right) & =\dot{\alpha_{n}(\theta)} \\
& \geq \quad \overline{\alpha_{n}}
\end{aligned}
$$

Hence, given any $\varepsilon>0$

$$
g^{(m(\theta))}(\theta, \psi(\theta))-\psi(\theta)-m(\theta)\left(\overline{\alpha_{n}}-\varepsilon\right) \quad>\quad 1
$$

This holds for all $\theta \in \mathbb{T}^{1}$, and so by Lemma 4.5 we have $\rho>\alpha_{n}^{-}-\varepsilon$. Since $\varepsilon$ was arbitrary, we must have $\rho \geq \dot{\alpha}$, as required. A similar argument gives $\rho \leq \alpha$. 
Of course, this is not particularly useful unless $\alpha_{n}^{+}$and $\alpha_{n}^{-}$converge to $\rho$ as $n \rightarrow \infty$. First note that for each $\theta \in \mathbb{T}^{1}, \alpha_{n}(\theta)$ and $\alpha_{n}^{+}(\theta)$ are bounded monotone sequences and hence converge to a limit. A similar argument to Lemma 4.5 gives

Lemma 5.2 If $\alpha_{n}^{+}$and $\alpha_{n}^{-}$are defined as above and $\rho$ is the rotation number of $F$, then

$$
\lim _{n \rightarrow \infty} \overline{\alpha_{n}}=\rho \quad=\quad \lim _{n \rightarrow \infty} \alpha_{n}^{+}
$$

Proof Given $\varepsilon>0$, for each $\theta \in \mathbb{T}^{1}$ we must have $\frac{1}{n}\left(g^{(n)}(\theta, \psi(\theta))-\psi(\theta)-1\right) \geq \rho-\varepsilon$ for arbitrarily large $n \in \mathbb{N}$. If not, so that $\frac{1}{n}\left(g^{(n)}(\theta, \psi(\theta))-\psi(\theta)-1\right)<\rho-\varepsilon$ for all sufficiently large $n$, we would have

$$
\lim _{n \rightarrow \infty} \frac{1}{n}\left(g^{(n)}(\theta, \psi(\theta))-\psi(\theta)-1\right) \quad \leq \quad \rho-\varepsilon
$$

and which contradicts the fact that the rotation number of $F$ is $\rho$. Choose $m(\theta)$ such that $m(\theta) \varepsilon>1$ and

$$
\frac{1}{m(\theta)}\left(g^{(m(\theta))}(\theta, \psi(\theta))-\psi(\theta)-1\right) \quad \geq \quad \rho-\varepsilon
$$

By Corollary 3.4 we have

$$
\begin{aligned}
\frac{1}{m(\theta)}\left(g^{(m(\theta))}(\theta, 0)-1\right) & \geq \quad \rho-\varepsilon-\frac{1}{m(\theta)} \\
& \geq \quad \rho-2 \varepsilon
\end{aligned}
$$

By continuity, for each $\theta \in \mathbb{T}^{1}$ choose an open interval $U(\theta) \subset \mathbb{T}^{1}$ such that

$$
\frac{1}{m(\theta)}\left(g^{(m(\theta))}\left(\theta^{\prime}, 0\right)-1\right) \quad \geq \quad \rho-3 \varepsilon
$$

for all $\theta^{\prime} \in U(\theta)$. Applying Corollary 3.4 again, we finally obtain

$$
\frac{1}{m(\theta)}\left(g^{(m(\theta))}\left(\theta^{\prime}, \psi\left(\theta^{\prime}\right)\right)-\psi\left(\theta^{\prime}\right)-1\right) \quad \geq \quad \rho-4 \varepsilon
$$

for all $\theta^{\prime} \in U(\theta)$. The $U(\theta)$ form an open cover of $\mathbb{T}^{1}$, so choose a finite subcover say $U\left(\theta_{1}\right), \ldots$, $U\left(\theta_{k}\right)$. For $\theta \in U\left(\theta_{k}\right)$ we have $\overline{\alpha_{n}}(\theta) \geq \rho-4 \varepsilon$ for any $n \geq m\left(\theta_{k}\right)$. Hence if we define $M=\max \{$ $\left.m\left(\theta_{1}\right), \ldots, m\left(\theta_{k}\right)\right\}$, then $\alpha_{n}(\theta) \geq \rho-4 \varepsilon$ for all $\theta \in \mathbb{T}^{1}$ for any $n \geq M$. Hence $\alpha_{n} \geq \rho-4 \varepsilon$ for all $n \geq M$ and so

$$
\lim _{n \rightarrow \infty} \overline{\alpha_{n}} \quad \geq \quad \rho-4 \varepsilon
$$

Since $\varepsilon$ is arbitrary, and by Lemma 5.1 we have $\overline{\alpha_{n}} \leq \rho$ for all $n$, this gives

$$
\lim _{n \rightarrow \infty} \overline{\alpha_{n}} \quad=\quad \rho
$$

as required. The argument for $\alpha_{n}^{+}$is identical.

As a corollary, we obtain the proof of Theorem 1.4. First note that since $\frac{1}{n}\left(g^{(n)}(\theta, y)-y\right)$ converges (to $\rho$ ) for any $(\theta, y) \in \mathbb{T}^{1} \times \mathbb{R}$, the sequence $\frac{1}{n}\left(g^{(n)}(\theta, \psi(\theta))-\psi(\theta)\right.$ ) is bounded for any $\theta \in \mathbb{T}^{1}$, and hence we may define 


$$
\alpha(\theta) \quad=\quad \sup _{n \in \mathbb{N}} \frac{1}{n}\left(g^{(n)}(\theta, \psi(\theta))-\psi(\theta)-1\right)
$$

Since $\alpha^{-}(\theta) \geq \alpha_{n}(\theta) \geq \dot{\alpha_{n}}$ for all $n$, we see that $\alpha^{-}(\theta)$ is bounded below and we may define

$$
\alpha \quad=\quad \inf _{\theta \in \mathbb{T}^{1}} \alpha(\theta)
$$

Similarly, we set

$$
\alpha^{+}(\theta) \quad=\quad \inf _{n \in \mathbb{N}} \frac{1}{n}\left(g^{(n)}(\theta, \psi(\theta))-\psi(\theta)+1\right)
$$

and

$$
\alpha^{+} \quad=\quad \sup _{\theta \in \mathbb{T}^{1}} \alpha^{+}(\theta)
$$

Given this notation, Theorem 1.4 can be stated as

Corollary 5.3 If $F$ has rotation number $\rho$, then

$$
\alpha=\rho \quad=\quad \alpha^{+}
$$

Proof By definition, we have $\alpha \leq \alpha(\theta)$ and

$$
\alpha(\theta) \quad=\quad \lim _{n \rightarrow \infty} \alpha_{n}(\theta)
$$

for all $\theta \in \mathbb{T}^{1}$. Given $\varepsilon>0$ there thus exists $N \in \mathbb{N}$ such that $\alpha(\theta)-\varepsilon \leq \alpha_{n}(\theta)$ for all $n \geq N$. Thus $\alpha-\varepsilon \leq$ $\alpha_{n}(\theta)$, and hence $\alpha-\varepsilon \leq \alpha_{n}$ for all $n \geq N$. Conversely, $\alpha(\theta) \geq \dot{\alpha_{n}}(\theta) \geq \alpha_{n}$ for all $n$ and thus $\alpha \geq \dot{\alpha}$ for all $n$. Thus $\dot{\alpha_{n}} \leq \alpha \leq \bar{\alpha}+\varepsilon$ for all $n \geq N$. By the previous lemma $\overline{\alpha_{n}} \rightarrow \rho$ as $n \rightarrow \infty$ and hence $\rho \leq \alpha \leq \rho+\varepsilon$. But $\varepsilon$ was arbitrary, and so $\rho=\alpha$, as required. The argument for $\alpha^{+}(\theta)$ is identical.

\section{ACKNOWLEDGEMENTS}

The early ideas in this paper were developed during a conference on Number Theory and Dynamical Systems held in York, UK in September 1997. JS and PAG could like to thank the conference organizers for their hospitality and the provision of such a stimulating environment and the London Mathematical Society for financial support. The attendance of JS at this conference was made possible by the UK EPSRC through grant GR/J97243. Further work was carried out under a British Council/DAAD academic exchange grant no. 774, and a Royal Society Leverhume Trust Senior Research Fellowship awarded to JS. JS would also like to gratefully thank the Institute of Physical Science and Technology at the U. of Maryland for their hospitality and financial support during two visits in 1998

\section{REFERENCES}

Arnold L, 1998, Random Dynamical Systems, Springer-Verlag, Berlin.

Arnold VI, 1957, Small Denominators I: Mappings of the Circumference to Itself, AMS Transl. Series 2, 46, 213-284. 
Arnold VI, 1983, Geometrical Methods in the Theory of Ordinary Differential Equations, Springer-Verlag, New York.

Chastell PR, Glendinning PA and Stark J, 1995, Determining the Locations of Bifurcations in Quasiperiodic Systems, Phys. Lett. A., 200, 17-26.

Derriennic Y and Krengel U, 1981, Subadditive Mean Ergodic Theorems, Ergod. Th. Dyn. Sys., 1, 33-48.

Ding M, Grebogi C and Ott E, 1989a, Dimensions of Strange Nonchaotic Attractors , Phys. Lett. A, 137, 167-172.

Ding M, Grebogi C and Ott E, 1989b, Evolution of Attractors in Quasiperiodically Forced Systems: From Quasiperiodic to Strange Nonchaotic to Chaotic, Phys. Rev. A, 39, 25932598.

Feudel U, Grebogi C and Ott E, 1997, Phase-locking in quasiperiodically forced systems., Phys. Rep., 290, 11-25.

Feudel U, Kurths J and Pikovsky A., 1995, Strange Non-chaotic Attractor in a Quasiperiodically Forced Circle Map, Physica D, 88, 176-186.

Glendinning P, Feudel U, Pikovsky AS and Stark J, 2000, The Structure of Mode-Locked Regions in Quasi-Periodically Forced Circle Maps, Physica D, 140, 227-243.

Herman MR, 1979, Sur la Conjugaison Différentiable des Difféomorphismes du Cercle à des Rotations, Publ. Math. IHES, 49, 5-233.

Herman MR, 1983, Une Méthode Pour Minorer les Exposants de Lyapunov et Quelques Examples Montrant le Caractère Local d'un Théorème d'Arnold et de Moser sur le Tore de Dimension 2, Comment. Math. Helvetici, 58, 453-502.

Katok A and Hasselblatt B, 1995, Introduction to the Modern Theory of Dynamical Systems, Cambridge University Press, Cambridge, UK.

Rhodes F and Thompson CL, 1986, Rotation Numbers for Monotone Functions of the Circle, J. Lond. Math. Soc., 34, 360-368.

Rhodes F and Thompson CL, 1991, Topologies and Rotation Numbers for Families of Monotone Functions on the Circle, J. Lond. Math. Soc., 43, 156-170.

Slomczynski W, 1995, Continuous subadditive processes and formulae for Lyapunov characteristic exponents, Annales Polonici Mathematici, 61, 101-134.

Slomczynski W, 1997, Subadditive Ergodic Theorems in $C(X)$, Italian Journal of Pure and Applied Mathematics, 1, 17-28.

Stark J, 1997, Invariant graphs for forced systems, Physica D, 109, 163-179.

Sturman R, 1999, Scaling of Intermittent Behaviour of a Strange Nonchaotic Attractor, Phys. Lett. A, 259, 355-365.

Sturman R , 2000, Strange Nonchaotic Attractors in Quasiperiodically Forced Systems, $\mathrm{PhD}$ Thesis, UCL.

Sturman R and Stark J, 2000, Semi-Uniform Ergodic Theorems and Applications to Forced Systems, Nonlinearity, 13, 113-143. 